Final Revised Manuscript (HMT_2017_83)

Accepted for publication in the International Journal of Heat and Mass Transfer

\title{
Computational/Analytical Study of the Transient Hot Wire-Based Thermal Conductivity Measurements near Phase Transition
}

\author{
Mahdi Nabil* and J. M. Khodadadi** \\ 1418 Wiggins Hall, Department of Mechanical Engineering \\ Auburn University, AL 36849-5341, USA
}

\begin{abstract}
The performance of the commonly-used transient hot wire technique for measuring the thermal conductivity of materials near their liquid-solid transition point is reported in this paper. Adopting a 1-D transient formulation, the computational methodology is discussed in detail and predictions of the wire temperature are compared to the limiting analytical solutions without and with phase change. By varying the initial solid-state temperature of an eicosane sample, six cases which undergo phase change to different extent were studied. The results exhibit a monotonic dependence of the predicted thermal conductivity value on the initial temperature of the solid medium. As the initial temperature of the solid-state sample approaches the melting point, the predicted thermal conductivity value moves toward the value of the liquid eicosane. Recommendations were provided for performing measurements of thermal conductivity using the transient how wire technique involving phase change.

* Graduate Research Assistant.

** $\quad$ Corresponding Author; Alumni Professor; Tel: 1(334) 844-3333; Fax: 1(334) 844-3307; khodajm@auburn.edu.
\end{abstract}

(C) 2017. This manuscript version is made available under the Elsevier user license http://www.elsevier.com/open-access/userlicense/1.0/ 


\section{KEY WORDS}

Eicosane; Enthalpy Method; Phase Transition; Thermal Conductivity Measurement; Transient Hot Wire Technique.

\section{$\underline{\text { Nomenclature }}$}

$\begin{array}{ll}a & \text { radius of the wire, } \mathrm{mm} \\ b & \text { radius of the cylindrical block, } \mathrm{mm} \\ C_{p} & \text { specific heat, } \mathrm{J} / \mathrm{kgK} \\ k & \text { thermal conductivity, } \mathrm{W} / \mathrm{mK} \\ L & \text { latent heat of fusion, } \mathrm{J} / \mathrm{kg} \\ q & \text { strength of the line heat source, } \mathrm{W} / \mathrm{m} \\ r & \text { radial coordinate, } \mathrm{mm} \\ s & \text { instantaneous position of the liquid-solid interface, } \mathrm{mm} \\ t & \text { time, } \mathrm{s} \\ T & \text { temperature, } \mathrm{K} \text { or }{ }^{\circ} \mathrm{C}\end{array}$

\section{Greek Symbols}

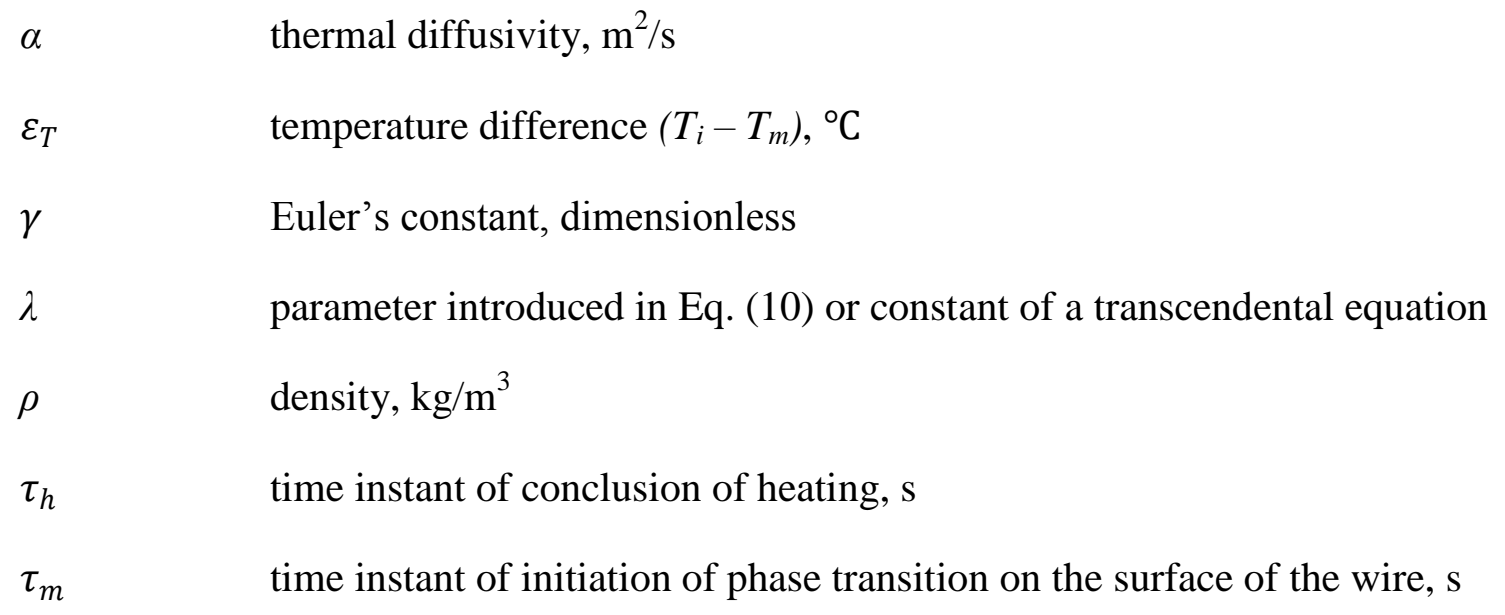

\section{Subscripts}

$i \quad$ initial


liquid

$m$

melting

S

solid

$w$

wire 


\section{Introduction}

Experimental techniques are widely used for determination of the transport properties of materials, such as thermal conductivity, viscosity, etc. These techniques are based on the fundamental relations that govern how the value of the pertinent flux (heat, momentum, etc.) relates to the gradient of the relevant quantity (temperature, velocity, etc.). For instance, both the steady-state and transient techniques that are used for determination of thermal conductivity utilize the Fourier's Law. The transient hot wire (THW) technique that is now widely used for determination of the thermal conductivity of gases, liquids, pastes, etc., has its roots in an ideal limiting case of a transient heat conduction problem (Carslaw and Jaeger [1]). In this formulation, an extremely thin wire of infinite length with a high thermal conductivity is surrounded by a homogeneous material that extends in the radial direction away from the wire to infinity. Upon heating the wire for a short time period, the transient signature of the wire's temperature is utilized to obtain the thermal conductivity of the surrounding material. The pertinent relation among the various governing parameters will be discussed below. Assael et al. [2] has discussed the historical evolution of the transient hot wire technique starting from 1780 up to 2010 .

Corrections to the transient hot wire technique that address deviations from the idealized formulation have also been developed. Nine (9) such modifications that account for effects of finite inner cylinder (heated wire), composite cylinders, the Knudsen effects, radiation, required condition at the outer cell circumference, the role of compressibility and natural convection, finite cell dimensions, variable fluid properties and the correction for finite length of wire were addressed in detail by Healy et al. [3]. Nagasaka and Nagashima [4] developed a new design of an instrument based on the transient hot wire technique by coating the platinum wire with a thin 
electrical insulation layer made of polyester 2. Through this modification, the capabilities of the instrument were broadened to include electrically-conducting fluids. Until that time, the bare metallic wire within the instrument was only compatible with electrically non-conducting media. The analytical solution considering the effects of the insulation layer on the temperature rise of the metallic wire was then discussed extensively. As a case study, thermal conductivity values of an aqueous $\mathrm{NaCl}$ solution was measured successfully with an accuracy of $\pm 0.5 \%$.

In recent years, operational deviations from the idealized case [1] have been treated computationally to include the above-mentioned or other effects, whereby analytical relations are replaced with numerical solutions of the governing differential equations, e.g. Assael et al. [5], Duluc et al. [6] and Rusconi et al. [7]. Assael et al. [5] used a numerical finite-element method (FEM) to solve a set of energy conservation equations which describe the operation of the transient hot wire technique. They showed that the adopted approach was applicable to predicting the thermal conductivity of liquids. The adopted approach considered the essential corrections to the ideal transient hot wire theoretical model which then led to accurate predictions when compared with the experimentally-measured thermal conductivity values. The authors presented computationally-obtained thermal conductivity values vs. measured quantities reported in the literature for Argon at high pressure conditions with an accuracy of $\pm 0.35 \%$. Utilizing the velocity-pressure formulation, spectral methods and domain decomposition technique, Duluc et al. [6] studied 2-D transient natural convection in liquid nitrogen around a pulse-heating thin bronze wire. The numerical data were then compared with their experimental observations. Considering time evolution of the wire's temperature rise, the theoretical heat conduction solution and numerical results were in close agreement during early time steps which indicated a purely conductive heat transfer regime. However, there was an onset time at which 
natural convection effects became important and thereafter results of the numerical simulation started to diverge from the experimental data. The authors proposed the wire thermal inertia as the key parameter that governs the aforementioned behavior. Rusconi et al. [7] studied the same phenomenon using the FlexPDE finite element method analysis of a 2-D cylindrical model. The Navier-Stokes equations along with the heat transport equation were solved simultaneously in the fluid region. A custom-built setup based on the transient hot wire method was also fabricated and the ensuing measurements of thermal conductivity were tested against results of the numerical simulation. Onset of natural convection effects after a specific time period was clearly observed. In order to exclude convection effects from the thermal conductivity measurements, an operational time scale was quantified. The suggested time-window depends on the dissipated power from the wire and the physical properties of the test fluid. It was also proved that the thickness and the physical properties of the wire insulation layer were such that it will not affect the obtained thermal conductivity values. Adoption of computational techniques to assess variations of the transient hot wire method has also been considered for other measurement techniques. For instance, Rusconi et al. [8] utilized computational fluid dynamics for refinement of thermal-convection effects in the thermal-lensing measurement technique.

Ongoing improvements, maturity and relevance of computational techniques that extend the range of applicability and account for actual physical and operating conditions of thermal conductivity measurements were outlined above. However, no studies that address issues in relation to the performance of the transient methods of measuring the thermal conductivity near the melting temperature of a medium were found. Therefore, computational modeling of the transient hot wire method and assessing its performance and operational issues while melting occurs during the thermal conductivity measurement is reported in this paper. 


\section{Problem Statement}

Measuring thermal conductivity of phase change materials (PCM) in their solid-state as a function of temperature using the transient methods, i.e. transient plane source (TPS) and transient hot wire methods, many researchers have studied thermal conductivity values as the reported measurement temperature approaches the melting point of the PCM. Recently, using the transient hot wire method, Wang et al. [9-12] reported experimentally-obtained thermal conductivity values for different phase change materials near their respective melting temperatures. A simple model of the typical reported dependence of thermal conductivity on temperature that assumes constant values of thermal conductivity of the liquid $\left(k_{l}\right)$ and solid $\left(k_{s}\right)$ phases near the melting temperature $\left(T_{m}\right)$ is shown in Figure 1. As the temperature of the measurement sample approaches the melting temperature, a sharp decline in the thermal conductivity values is registered. Operation of the THW technique under such non-equilibrium condition is not consistent with the idealized theory [1] based on which the transient techniques of measuring thermal conductivity are designed to be applicable. Moreover, the THW technique is ideally-suited to measure the thermal conductivity of a homogenous medium. Therefore, once these conditions break down and the solid PCM starts transforming into two separate phases, the measured thermal conductivity values are not accurate any longer. In this paper, issues related to measuring the thermal conductivity of phase change materials near their melting temperatures are addressed through adopting both analytical and computational methodologies.

The geometry of the model problem consists of a solid cylindrical block of radius $b$ that includes a wire heat source of radius a positioned along the symmetry axis of the block. The idealized theories consider a block that extends to infinity and surrounds a zero-thickness wire as shown in Figure 2, whereas using a computational approach and given the finite size of actual 
THW cells necessitates adoption of a finite radius for the block. The model block is insulated on the top and bottom planes $(z=$ constant $)$, whereas the outer radial boundary of the medium remains at the initial temperature $T_{i}$ of the system (that is below the melting temperature by a very small amount $\varepsilon_{T}=T_{m}-T_{i}$ ). Also note that the initial temperature $T_{i}$ is the temperature at which the measured thermal conductivity is reported. For time $t>0$, thermal energy is released within the highly conductive wire, thus raising its temperature rather evenly. Depending on the initial temperature and properties of the wire and PCM, a liquid layer might form next to the wire after a time delay $\left(\tau_{m}\right)$. Under this condition, the instantaneous liquid-solid interface is at the radial coordinate $s(t)$ that moves away from the wire as time increases (Figure 2).

\section{Governing Equations}

It was assumed that the solid heating wire and the PCM surrounding it in both solid and liquid phases are homogenous and isotropic. The one-dimensional (1-D) transient melting process that takes place under condition of cylindrical axisymmetry is governed by heat conduction. Densities of the liquid and solid phases of PCM are generally not equal. However, in this paper the density variation is ignored. The thermal energy equations for the two-phase medium which need to be solved in order to obtain the temperature distributions in the liquid and solid phases are as follows.

Subscripts $s, l$ and $w$ are adopted for the solid phase, liquid phase and wire, respectively.

Within the solid layer of the medium, i.e. for $a<r<b$ and $t>0$ :

$$
\left(\rho C_{p}\right)_{s} \frac{\partial T_{S}}{\partial t}=k_{s}\left(\frac{1}{r} \frac{\partial T_{S}}{\partial r}+\frac{\partial^{2} T_{s}}{\partial r^{2}}\right)
$$


For the liquid layer formed between the wire and the solid-liquid interface, i.e. for $a \leq r<$ $s(t)$ and $t \geq \tau_{m}$

$$
\left(\rho C_{p}\right)_{l} \frac{\partial T_{l}}{\partial t}=k_{l}\left(\frac{1}{r} \frac{\partial T_{l}}{\partial r}+\frac{\partial^{2} T_{l}}{\partial r^{2}}\right) .
$$

The thermal energy equation for the wire that is valid for $0<r \leq a$ and $t>0$, with the constant heat source of $q\left[\frac{W}{m}\right]$ is:

$$
\left(\rho C_{p}\right)_{w} \frac{\partial T_{w}}{\partial t}=k_{w}\left(\frac{1}{r} \frac{\partial T_{w}}{\partial r}+\frac{\partial^{2} T_{w}}{\partial r^{2}}\right)+\frac{q}{\pi a^{2}},
$$

where the symbols $k, \rho$ and $C_{p}$ denote the thermal conductivity, density and specific heat, respectively. Relations 1-3 are analogous to those of Assael et al. [5] except for the introduction of the conduction equation for the liquid phase.

At the liquid/solid interface, i.e. for $r=s(t) \geq a$ and $t \geq \tau_{m}$,

$$
k_{s} \frac{\partial T_{s}}{\partial r}-k_{l} \frac{\partial T_{l}}{\partial r}=\rho L \frac{d s(t)}{d t},
$$

where $L$ stands for the heat of fusion. The set of equations above is subject to the initial and boundary conditions as follows:

For $t=0$ and $r>0$

$$
T_{w}=T_{s}=T_{i}
$$

For $t>0$ at $r=0$, due to symmetry,

$$
\frac{\partial T_{w}}{\partial r}=0
$$

For $t>0$ at $r=b$,

$T_{s}=T_{i}$

For $t \geq \tau_{m}$ at $r=s(t)$,

$$
T_{s}(r, t)=T_{l}(r, t)=T_{m}
$$


For $0<t<\tau_{m}$ at $r=a$

For $t \geq \tau_{m}$ at $r=a$,

$$
\begin{aligned}
& k_{w}\left(\frac{\partial T_{w}}{\partial r}\right)_{r=a}=k_{s}\left(\frac{\partial \mathrm{T}_{\mathrm{s}}}{\partial r}\right)_{r=a} \\
& \text { and } \quad T_{w}(a, t)=T_{s}(a, t) \text {. } \\
& k_{w}\left(\frac{\partial T_{w}}{\partial r}\right)_{r=a}=k_{l}\left(\frac{\partial \mathrm{T}_{l}}{\partial r}\right)_{r=a} \\
& \text { and } \quad T_{w}(a, t)=T_{l}(a, t) \text {. }
\end{aligned}
$$

\section{Computational Methodology}

\subsection{Modeling of the commonly-used THW technique using ANSYS ${ }^{\circledR}$ FLUENT}

A 1-D transient axisymmetric computational model for the THW technique is utilized in this study. The governing heat conduction equations in combination with a phase change enthalpy model were solved using the ANSYS ${ }^{\circledR}$ FLUENT software. A solid eicosane cylindrical block was chosen as a case study with a platinum wire heat source positioned along the symmetry axis of the block. The adopted thermo-physical properties of eicosane and platinum from the literature are given in Table 1 and Table 2, respectively. The solution domain was insulated on the top and bottom planes, whereas the outer radial boundary of the medium remained at the initial temperature $T_{i}$. At $t=0$, the solid block is at a uniform temperature $T_{i}$ (below the melting temperature by a very small amount $\varepsilon_{T}=T_{m}-T_{i}$ ). Thermal energy is then released within the wire at a constant heating power for a specific time period and thereafter the power supply is turned off (pulse function). The amount of heating power and the heating

time duration, i.e. $1 \frac{W}{m}$ and 1 second, respectively, were chosen in accordance with practices of actual THW experimental measurement conditions reported by Assael et al. [5]. At the end of the heating period, the temperature rise on the surface of the wire $(\Delta T(a, t))$ is plotted against 
the heating time on a semi-logarithmic scale. The thermal conductivity value of the PCM is then extracted from the slope of the $\Delta T(a, t)$ versus time curve, in accordance with the following equation given by Carslaw and Jaeger [1] for the idealized THW technique:

$$
\Delta T(a, t)=\frac{q}{4 \pi k} \ln \left(\frac{4 \alpha t}{a^{2} c}\right)
$$

where $\alpha, k$ and $a$ represent the thermal diffusivity of the PCM, thermal conductivity of the PCM and radius of the platinum wire, respectively. Furthermore, $q$ is the constant power of the heat source per unit length of the wire, $C=\exp (\gamma)=1.7811$, and $\gamma$ is the Euler's constant. The slope of the $\Delta T(a, t)$ versus time curve is equal to $\frac{q}{4 \pi k}$, allowing for extraction of the value of thermal conductivity. The procedure is explained extensively by de Groot et al. [19], Healy et al. [3] and Roder [20].

An enthalpy method incorporated within version 13 of the commercial CFD code ANSYS $^{\circledR}$ FLUENT was used to solve the governing equations. The equations were solved for $1 \mathrm{sec}$ with the computational time step equal to $10 \mu \mathrm{s}$. The adopted values of the underrelaxation factors for the liquid fraction and thermal energy were 0.9 and 1 , respectively. In order to satisfy the convergence criterion $\left(10^{-9}\right.$ for thermal energy equation), the maximum number of iterations for each time step was set to 200. However, the required number of iterations to satisfy the convergence criterion at each time step was observed to be between 10 and 20. The wire and the solid medium were discretized in the radial direction. Considering the cylindrical coordinate system, the computational domain had a length of $6 \mathrm{~mm}$ along the radial direction and $1 \mathrm{~mm}$ height in the $z$-direction. Since a 1-D conduction problem was only studied here, the height of the domain had no influence on the results. The finite axial thickness was only chosen to allow for visual tracking of the liquid/solid interface movement during the 
simulation time period. The wire region of length $8 \mu \mathrm{m}$ consisted of 20 elements and the solid region of length $5.992 \mathrm{~mm}$ containing the PCM was divided into 230 elements, while the spacings of both groups of elements were non-uniform. The geometric expansion ratios used for laying the non-uniform grids within the wire and solid medium were 1.1 and 1.02 , respectively. The aim was to create more dense mesh arrangements within each region on both sides of the wire surface. In the $z$-direction, the height of both wire and solid domains were divided uniformly into 3 equal elements.

\subsection{Exact solution of an ideal melting problem}

An exact solution for melting of an infinite domain that contains a zero-thickness heating wire is derived by Paterson [21], hereto called the Paterson Model. Formulation for this problem and its solution were repeated with the notations for the relevant quantities matching the related solidification (freezing) problem described by Özişik [22]. The pertinent equations were coded and solved with the aid of the MATLAB software. The objective was to verify the accuracy of predictions of the FLUENT model in the case that phase change occurs. Having discussed the adopted analytical and numerical models, a summary of the various aspects of the models are

given in Table 3. It is clear that among the three methods, the computational approach is capable of handling the realistic operating conditions of the THW-based thermal conductivity measurements.

\section{Results and Discussion}

\subsection{Validation of the FLUENT model against the ideal THW theory (without phase change)}


The mathematical formulation by Carslaw and Jaeger [1], i.e. equation (6) for prediction of the temperature rise on the surface of the wire of the transient hot wire technique assumes an ideal perfect wire conductor that is surrounded by an infinite medium. An attempt was made to verify the accuracy of the results of the adopted ANSYS ${ }^{\circledR}$ FLUENT model versus the ideal limiting-case model of Carslaw and Jaeger [1]. To achieve this, the value of thermal conductivity of the heating wire was set to a very large number, i.e. 1000 times the thermal conductivity of platinum $(71.7 \mathrm{~W} / \mathrm{mK}[16])$. For the next case study, the actual thermal conductivity value of platinum wire was assigned. The ideal theory is only applicable for homogenous media in which phase transition does not occur during the heating period. Thus, the initial temperature was selected as $T_{i}=306 \mathrm{~K}$ to ensure that the final temperature will not reach the melting point of eicosane, i.e. $T_{m}=310 \mathrm{~K}$. The FLUENT-based temperature rise versus time results are compared to the predictions of the ideal Carslaw and Jaeger [1] model in Figure 3. Comparing the temperature difference of each FLUENT case with the prediction of the ideal model at $t=1 \mathrm{~s}$, there are $2.17 \%$ and $0.01 \%$ errors corresponding to wire thermal conductivities of 71.7 and $71700 \mathrm{~W} / \mathrm{mK}$, respectively. This confirmed that as the adopted thermal conductivity value of the heating wire approaches a very large value which theoretically is called a perfect conductor, the temperature rise behavior is very similar to the prediction based on the model of Carslaw and Jaeger [1]. It was further observed that the FLUENT-predicted average temperature rise within the heating wire was very close to the amount of the temperature rise on the wire surface.

\subsection{Validation of the FLUENT model against the Paterson's melting problem}

Paterson [21] derived an exact solution for melting of an infinite domain due to a zerothickness line heat source of strength $q$ positioned at $r=0$. The solid body is initially at a 
uniform temperature $T_{i}$ lower than the melting point $\left(T_{m}\right)$ of the medium. Thereafter, the heat source is turned on to continuously release thermal energy. Consequently, melting begins at $r=0$ and the liquid/solid interface starts to move in the radial direction. The analytical model of Paterson [21] predicts the instantaneous temperatures for both the liquid and solid domains and also the location of the solid/liquid interface at any time instant. The instantaneous temperature distributions within the liquid and solid domains are:

$$
\begin{array}{ll}
T_{l}(r, t)=T_{m}+\frac{q}{4 \pi k_{l}}\left[-E i\left(-\frac{r^{2}}{4 \alpha_{l} t}\right)+E i\left(-\lambda^{2}\right)\right] & \text { for } \quad 0<r \leq s(t), \\
T_{S}(r, t)=T_{i}-\frac{T_{i}-T_{m}}{E i\left(-\lambda^{2} \alpha_{l} / \alpha_{s}\right)} E i\left(-\frac{r^{2}}{4 \alpha_{s} t}\right) & \text { for } \quad s(t) \leq r<\infty,
\end{array}
$$

where the exponential-integral function is defined as (Abramowitz and Stegun [23]):

$$
-E i(-x) \equiv E_{1}(x)=\int_{x}^{\infty} \frac{e^{-u}}{u} d u=\int_{1}^{\infty} \frac{e^{-x t}}{t} d t \quad \text { for } \quad x>0 .
$$

The location of the liquid/solid interface will be determined through:

$$
s(t)=2 \lambda\left(\alpha_{l} t\right)^{1 / 2},
$$

where $\lambda$ is a positive constant that is calculated by solving the following transcendental equation [21]:

$$
\frac{q}{4 \pi} e^{-\lambda^{2}}+\frac{k_{s}\left(T_{m}-T_{i}\right)}{E i\left(-\lambda^{2} \alpha_{l} / \alpha_{s}\right)} e^{-\lambda^{2} \alpha_{l} / \alpha_{s}}=\lambda^{2} \alpha_{l} \rho L
$$

Once $\lambda$ is obtained, the location of the liquid/solid interface can easily be tracked versus time using eq. (10). Furthermore, the temperature distributions within both the liquid and solid regions will also be known through equations (7) and (8). 
In this study, a line heat source of strength $q=1 \mathrm{~W} / \mathrm{m}$ was used in accordance with operation of the commonly-used THW technique (Assael et al. [5]). Solid eicosane was initially at a uniform temperature $T_{i}=309.9 \mathrm{~K}$ that is a bit lower than its melting point $\left(T_{m}=310 \mathrm{~K}\right)$. Figure 4(a) exhibits the variation of the relevant transcendental function (eq. 11) as a function of $\lambda$. Using the Newton-Raphson root-finding algorithm (Quarteroni et al. [24]) for solving the transcendental equation 11, the pertinent value of $\lambda$ was found. Knowing the value of $\lambda$, the instantaneous location of the liquid/solid interface based on Eq. (10) is shown in Figure 4(b). Temperature distributions within the liquid and solid domains as a function of time and location are exhibited in Figure 5(a). The solid black line in the figure is the instantaneous location of the liquid/solid interface. In order to verify the accuracy of the FLUENT model against the analytical solution of Paterson [21] under phase change condition, a case study was run with the same initial and boundary conditions using ANSYS ${ }^{\circledR}$ FLUENT. Variations of the temperature rise on the surface of the wire which are obtained from the Paterson [21] and FLUENT models versus heating time (i.e. $1 \mathrm{sec}$ ) are shown in Figure 5(b). Since $r=0$ is a singular point in the model of Paterson [21], three other position at $r=2,2.5$ and $3 \mu \mathrm{m}$ were chosen as the surrogate "locations on the surface of the wire" for obtaining the temperature rise data. The trends of the temperature rise curves predicted by both models (Fig. 5(b)) are very similar as seen in the inset box and the observed deviations are less than $0.5 \%$.

So far, validation of the FLUENT model was compared against idealized models without [1] and with phase change [21]. Focus is now placed on case studies for which the FLUENT model is used for predicting the values of thermal conductivity of PCM. In effect, the most general case involving various dimensionless groupings listed in the bottom row of Table 3 along with a finite-size wire and finite medium domain are considered. 


\subsection{Thermal conductivity predictions using the FLUENT model}

\subsubsection{Two validation case studies without phase change}

In the first of these two case studies, solid eicosane that was initially at a uniform temperature of $T_{i}=306 \mathrm{~K}$ (lower than the melting temperature by $4{ }^{\circ} \mathrm{C}$ ) was continuously heated for $1 \mathrm{sec}$. The initial temperature was selected such that the final temperature on the wire surface will be lower than the melting point. The initially-assigned thermal conductivity value for eicosane in the solid phase was set to $k_{s}=0.42 \frac{W}{m K}$ (Stryker and Sparrow [13]). The corresponding temperature rise $(\Delta T)$ on the wire surface versus time is shown in Figure 6. In plotting this figure, ten (10) temperature rise data points on each of the two ends were eliminated as suggested by de Groot et al. [19]. In effect, the temperature rise data did not begin at its initial value of zero. Discarding of these data points did not affect the value of the slope of the curve fit that was used to extract the value of thermal conductivity. A similar procedure was applied to all subsequent temperature rise versus time plots that will be presented. Using Eq. (6) due to Carslaw and Jaeger [1], the predicted thermal conductivity value based on the slope the leastsquares curve fit to the FLUENT data of Figure 6 was equal to $\left(k_{s}\right)_{\text {predicted }}=0.4242 \frac{\mathrm{W}}{\mathrm{mK}}$. Therefore, the extracted thermal conductivity value based on the FLUENT model prediction is only off from the assigned value by $1 \%$. In a similar case study, liquid eicosane initially at a uniform temperature of $T_{i}=313 \mathrm{~K}$ (higher than the melting temperature by $3{ }^{\circ} \mathrm{C}$ ) was heated for $1 \mathrm{sec}$. The initially-assigned thermal conductivity value for eicosane in the liquid state was set to $k_{l}=0.148 \frac{W}{m K}$ (Yaws [15]). The associated variation of the temperature rise $(\Delta T)$ on the wire surface versus time is presented in Figure 7. Using Eq. (6), the predicted thermal conductivity value based on the slope of the least-squares curve fit of Figure 7 was equal to 
$\left(k_{l}\right)_{\text {predicted }}=0.1468 \frac{\mathrm{W}}{\mathrm{mK}}$. Therefore, the extracted thermal conductivity value based on the prediction of the FLUENT model deviates from the assigned value by only $0.81 \%$.

Finally, note that the values of the $R^{2}$ coefficients of determination of the least-squares curve fits of Figures 6 and 7 (cases without phase transition) were equal to unity. It needs to be reiterated that the $\Delta T$ curves of Figures 6 and 7 are the observed temperature rise on the wire surface $(r=a)$. With the thermal diffusivity of eicosane in the liquid state lower than that of the solid state by a factor of 3.64 , the released thermal wave from the wire did not penetrate into the liquid medium (Fig. 7) surrounding the wire as fast as the solid domain (Fig. 6). In effect, during the monitored heating time period (same as temperature recording period) for the case of the liquid medium surrounding the wire, a greater portion of the total heat source increased the temperature of the wire itself which is observed on the wire surface.

\subsubsection{Effect of the initial solid-state temperature on the predicted thermal conductivity value with} phase change

As discussed earlier, in using the transient hot wire technique, many researchers have reported their measurements of the thermal conductivity values as the measurement temperature approaches the melting point of the PCM (e.g. Wang et al. [9-12]). In this section, the effect of the initial temperature of the medium on the measured thermal conductivity near the melting temperature is studied through adoption of the FLUENT model.

Six (6) cases with different solid-state initial temperatures were studied. The initial temperatures were selected in order to assure occurrence of phase transition during the heating time period (i.e. $1 \mathrm{sec}$ ). The selected solid-state initial temperatures were equal to $T_{i}=$ $308.3,308.4,308.5,308.6,308.7,308.9 \mathrm{~K}$, corresponding to $\varepsilon_{T}=1.7,1.6,1.5,1.4,1.3,1.1{ }^{\circ} \mathrm{C}$, 
respectively. Variations of the temperature on the surface of the wire versus heating time for all cases are shown in Figure 8. The temperature on the surface of the wire corresponding to the lowest initial temperature considered $(308.3 \mathrm{~K})$ exhibits a monotonic rise during the heating period and no liquid PCM is sensed on the surface of the wire. On the other hand, as the initial temperature is raised, the observed temperature on the surface of the wire exhibits two distinct trends. Firstly, the monotonic temperature rise portion of the heating period is shortened and liquid PCM is observed on the surface of the wire at an earlier time instant within the heating period. Secondly, upon formation of a less conductive liquid layer (also lower thermal diffusivity) surrounding the wire, the temperature on the surface of the wire rises faster as reasoned earlier for no phase change cases of Figures 6 and 7. Following the procedure discussed earlier, the predicted "effective" thermal conductivity values corresponding to various initial temperatures are given in Table 4 and exhibited schematically in Figure 9. Furthermore, the corresponding plots of the temperature rise $(\Delta T)$ on the wire surface versus the heating time along with the respective least-squares curve fits are shown for these six cases in Figures 10-15. For initial solid-state temperature values between 308.4 and $308.9 \mathrm{~K}$, the recorded temperature rise curves exhibit a variety of trends and these variations exhibit great deviations from the no phase change cases of Figures 6 and 7 that are ideally intended for THW measurements under equilibrium conditions. Excluding the lowest initial temperature, the derived "effective thermal conductivity" values are lower than the assigned thermal conductivity of the solid-state value $(0.42 \mathrm{~W} / \mathrm{mK})$ by $1.19-64.62 \%$ exhibiting an inverse relation with the initial solid-state temperature. Except the case of the lowest initial solid-state temperature $\left(T_{i}=308.3 \mathrm{~K}\right)$, the values of the $R^{2}$ coefficients of determination of the least-squares curve fits of Figures 11-15 ranged between $0.9215-0.9675$ signifying deterioration of the quality of the curves fits in 
comparison to those of Figs. 6, 7 and 10. For the lowest initial solid-state temperature, i.e. $T_{i}=308.3 \mathrm{~K}$, phase change occurs at the very end of the heating period and the predicted thermal conductivity value is almost equal to the solid-state value. In order to explain the observed relation between the initial solid-state temperature and the predicted "effective" thermal conductivity values, note that to start with, there is a composite system that contains the wire and the solid PCM. Upon heating of the wire, a portion of the diffused thermal energy away from the wire turns the solid possessing a high value of thermal conductivity $(0.42 \mathrm{~W} / \mathrm{mK})$ next to the wire into liquid that has a lower thermal conductivity $(0.148 \mathrm{~W} / \mathrm{mK})$. Following the argument above in relation to cases without phase transition (Figs. 6 and 7), less heat diffuses in the radial direction through a less-conductive (also lower thermal diffusivity) thickening liquid layer, and a greater portion of the total heat source fails to reach the solid region. The effective thermal conductivity of the composite is observed to be a value between the thermal conductivity values of the liquid and solid phases. This predicted value is closer to the thermal conductivity of the liquid state as the initial solid-state temperature approaches the melting temperature of the PCM.

\section{$\underline{5.3 .3 \text { Recommendations for THW experiments near phase transition }}$}

Based on the above discussions, it is clear that accurate measurements of thermal conductivity of materials in their solid state near phase transition require extra care. Firstly, in the planning stage of the experiments, one should assure that combination of the operating conditions and materials properties will not give rise to extensive formation of a liquid layer next to the heated wire. Defining the heating time period of the wire to be $\tau_{h}$, Eq. 6 [1] can be used to come up with the following criterion: 


$$
\varepsilon_{T} \geq \frac{q}{4 \pi k_{s}} \ln \left(\frac{4 \alpha \tau_{h}}{a^{2} C}\right)
$$

This establishes an explicit relation among the strength of line heat source $(q)$, temperature difference $\left(\varepsilon_{T}\right)$ and heating time period $\left(\tau_{h}\right)$ that the user can tailor for the application at hand in order to avoid excessive forming of a liquid layer next to the heated wire. In applying this relation to the cases of Figures $10-15$ (i.e. $\varepsilon_{T}=1.7-1.1{ }^{\circ} \mathrm{C}$ ), Eq. (12) becomes $\varepsilon_{T} \geq 1.73{ }^{\circ} \mathrm{C}$. This finding is in excellent agreement with the temperature rise data of Figure 8 since the only case with the minimum amount of liquid formation next to the wire corresponded to the case of $\varepsilon_{T}=1.7{ }^{\circ} \mathrm{C}$ (Fig. 10). A similar criterion can be obtained by enforcing the same condition to the theoretical relations of Paterson [21] leading to the following requirement:

$$
\frac{E i\left(-a^{2} / 4 \alpha_{s} \tau_{h}\right)}{E i\left(-\lambda^{2} \alpha_{l} / \alpha_{s}\right)} \leq 1
$$

This approach is far more involved compared to the explicit relation 12.

Based on the temperature rise curves discussed earlier, the user is also recommended to check the values of the $R^{2}$ coefficients of determination of the least-squares curve fits. Further deviation of this coefficient from unity as observed for Figures 11-15 and summarized in Table 4 can be used a simple measure of the reliability of the "effective" thermal conductivity. Finally, it should be noted that even though the present work was focused specifically on the transient hot wire technique, the methodology can be extended to other transient techniques such as the transient plane source (TPS) method that have been used for thermal conductivity determination of nanostructure-modified phase change materials $[25,26]$.

\section{Conclusions}


With the aid of the FLUENT software, a 1-D transient conduction problem was formulated and solved over a finite cylindrical domain without and with phase transition. The goal was to model the performance of the THW technique near the melting temperature of the medium. The model was first successfully verified against the ideal transient hot wire theory for a perfect conductor heat source with a $0.01 \%$ error in the values of monitored temperatures. Another validation was performed against an analytical solution for a model melting problem to verify the adopted enthalpy method in the FLUENT model. The registered error for the monitored temperatures was less than $0.5 \%$. Thereafter, focusing on the FLUENT model, thermal conductivity predictions in both liquid and solid phases were performed in the absence of phase change. The difference between the extracted thermal conductivity values and the initially-assigned values was less than $1 \%$ and $0.81 \%$ for solid and liquid phases, respectively. Finally, the effect of the initial solid-state temperature on the predicted value of thermal conductivity with phase change was explored. The predicted thermal conductivity values were lower than the assigned thermal conductivity of the solid-state value by $1.19-64.62 \%$, exhibiting an inverse relation with the initial solid-state temperature. Recommendations were provided for performing measurements of thermal conductivity using the transient how wire technique near phase transition.

\section{Acknowledgement}

This material is based upon work supported by the US Department of Energy under Award Number DE-SC0002470 (http://www.eng.auburn.edu/nepcm). The first author acknowledges the Department of Mechanical Engineering and the Samuel Ginn College of Engineering at Auburn University for partial support of his College of Engineering Graduate 
Fellowship. The second author acknowledges that the research leading to this publication has received funding from the European Union's Seventh Framework Programme (FP7/2007-2013) under grant agreement no. PIRSES-GA-2013-610692 (INNOSTORAGE; http://www.innostorage.eu/). 


\section{$\underline{\text { References }}$}

[1] Carslaw, H. S., and Jaeger, J. C., 1959, Conduction of Heat in Solids, $2^{\text {nd }}$ edition, Oxford University Press, London, UK.

[2] Assael, M. J., Antoniadis, K. D., and Wakeham, W. A., 2010, "Historical Evolution of the Transient Hot-Wire Technique," International Journal of Thermophysics, 31, pp. 10511072.

[3] Healy, J. J., de Groot, J. J., and Kestin, J., 1976, "The Theory of the Transient Hot-Wire Method for Measuring Thermal Conductivity," Physica, 82C, pp. 392-408.

[4] Nagasaka, Y., and Nagashima, A., 1981, "Absolute Measurement of the Thermal Conductivity of Electrically Conducting Liquids by the Transient Hot-Wire Method," Journal of Physics E: Scientific Instruments, 14, pp. 1435-1440.

[5] Assael, M. J., Karagiannidis, L., Malamataris, N., and Wakeham, W. A., 1998, "The Transient Hot-Wire Technique: A Numerical Approach," International Journal of Thermophysics, 19, pp. 379-389.

[6] Duluc, M-C, Xin, S., and Le Quere, P., 2003, "Transient Natural Convection and Conjugate Transients around a Line Heat Source," International Journal of Heat and Mass Transfer, 46, pp. 341-354.

[7] Rusconi, R., Williams, W. C., Buongiorno, J., Piazza, R., and Hu, L-W, 2007, "Numerical Analysis of Convective Instabilities in A Transient Short-Hot-Wire Setup for Measurement of Liquid Thermal Conductivity," International Journal of Thermophysics, 28(4), pp. 1131-1146.

[8] Rusconi, R., Isa, L., and Piazza, R., 2004, "Thermal-Lensing Measurement of Particle Thermophoresis in Aqueous Dispersions," Journal of the Optical Society of America B, 21(3), pp. 605-616.

[9] Wang, J., Xie, H., and Xin, Z., 2008, “Thermal Properties of Heat Storage Composites Containing Multiwalled Carbon Nanotubes," Journal of Applied Physics, 104, 113537, 5 pages.

[10] Wang, J., Xie, H., and Xin, Z., 2009, "Thermal Properties of Paraffin Based Composites Containing Multi-Walled Carbon Nanotubes," Thermochimica Acta, 488, pp. 39-42.

[11] Wang, J., Xie, H., Li, Y., and Xin, Z., 2010a, "PW Based Phase Change Nanocomposites Containing $\gamma-\mathrm{Al}_{2} \mathrm{O}_{3}$," Journal of Thermal Analysis and Calorimetry, 102(2), pp. 709-713. 
[12] Wang, J., Xie, H., Xin, Z., Li, Y., and Chen, L., 2010b, "Enhancing Thermal Conductivity of Palmitic Acid Based Phase Change Materials with Carbon Nanotubes as Fillers," Solar Energy, 84, pp. 339-344.

[13] Stryker, P. C., and Sparrow, E. M., 1990, "Application of a Spherical Thermal Conductivity Cell to Solid n-Eicosane Paraffin," International Journal of Heat and Mass Transfer, 33, pp. 1781-1793.

[14] Humphries, W. R., and Griggs, E. I., 1977, A Design Handbook for Phase Change Thermal Control and Energy Storage Devices, National Aeronautics and Space Administration (NASA), Huntsville, AL, USA.

[15] Yaws, C. L., 1995, Handbook of Thermal Conductivity, Volume 3: Organic Compounds $C_{8}$ to $C_{28}$, Gulf Professional Publishing, Houston, TX, USA.

[16] Hale, D. V., Hoover, M. J., and O’Neill, M. J., 1971, Phase Change Materials Handbook, National Aeronautics and Space Administration (NASA), Huntsville, AL, USA.

[17] Grigoriev, I. S., and Meilikhov, E. Z., 1997, Handbook of Physical Quantities, CRC-Press LLC, Boca Raton, FL, USA.

[18] Goldsmith, A., Waterman, T. E., and Hirschhorn, H. J., 1961, Handbook of Thermophysical Properties of Solid Materials, Volume 1: Elements, The MacMillan Company, New York, NY, USA.

[19] de Groot, J. J., Kestin, J., and Sookiazian, H., 1974, "Instrument to Measure the Thermal Conductivity of Gases," Physica, 75, pp. 454-482.

[20] Roder, H. M., 1981, “A Transient Hot Wire Thermal Conductivity Apparatus for Fluids,” Journal of Research of the National Bureau of Standards, 86, pp. 457-493.

[21] Paterson, S., 1952, "Propagation of a Boundary of Fusion," Proceedings of the Glasgow Mathematical Association, 1, pp. 42-47.

[22] Özişik, M. N., 1993, Heat Conduction, $2^{\text {nd }}$ Edition, John Wiley \& Sons Inc., New York, NY, pp. 408-412.

[23] Abramowitz, M., and Stegun, I. A., 1964, Handbook of Mathematical Functions, National Bureau of Standards (NBS) Applied Mathematic Series, 55, U.S. Government Printing Office, Washington, D.C., pp. 228-229.

[24] Quarteroni, A., Sacco, R., and Saleri, F., 2007, Numerical Mathematics, Springer, Berlin, Germany. 
[25] Nabil, M., and Khodadadi, J. M., 2013, "Experimental Determination of TemperatureDependent Thermal Conductivity of Solid Eicosane-Based Nanostructure-Enhanced Phase Change Materials," International Journal of Heat and Mass Transfer, 67, pp. 301-310.

[26] Al Ghossein, R. M., Hossain, M. S. and Khodadadi, J. M., 2017, "Experimental Determination of Temperature-Dependent Thermal Conductivity of Solid Eicosane-Based Silver Nanostructure-Enhanced Phase Change Materials for Thermal Energy Storage," International Journal of Heat and Mass Transfer, 107, pp. 697-711. 


\section{List of Tables}

Table 1 Thermo-physical properties of eicosane $\left(\mathrm{C}_{20} \mathrm{H}_{42}\right)$ adopted from the literature

Table 2 Thermo-physical properties of platinum ( $\mathrm{Pt}$ ) adopted from the literature

Table 3 Specifications of the adopted analytical/computational methodologies

Table 4 Predicted thermal conductivity data versus the initial solid-state temperature of eicosane using the FLUENT model 
Table 1 Thermo-physical properties of eicosane $\left(\mathrm{C}_{20} \mathrm{H}_{42}\right)$ adopted from the literature

\begin{tabular}{|c|c|}
\hline Property (Reference) & Value \\
\hline $\begin{array}{c}\rho\left(\mathrm{kg} / \mathrm{m}^{3}\right) \\
\text { (Stryker and Sparrow [13]) }\end{array}$ & 840 \\
\hline $\begin{array}{c}\left(C_{p}\right)_{s} \text { and }\left(C_{p}\right)_{l}(\mathrm{~J} / \mathrm{kgK}) \\
(\text { Humphries and Griggs [14]) }\end{array}$ & 1,920 and 2,460 \\
\hline $\begin{array}{c}k_{s} \text { and } k_{l}(W / m K) \\
\text { (Stryker and Sparrow [13]) } \\
\text { (Yaws [15]) }\end{array}$ & 0.42 and 0.148 \\
\hline $\begin{array}{c}\quad L(J / k g) \\
(\text { Hale et al. }[16])\end{array}$ & 247,000 \\
\hline $\begin{array}{c}T_{m}(K) \\
\text { (Hale et al. [16]) }\end{array}$ & 310 \\
\hline
\end{tabular}


Table 2 Thermo-physical properties of platinum (Pt) adopted from the literature

\begin{tabular}{|c|c|}
\hline Property (Reference) & Value \\
\hline$\rho\left(\mathrm{kg} / \mathrm{m}^{3}\right)$ & 21,450 \\
\hline (Grigoriev and Meilikhov [17]) & \\
\hline$C_{p}(\mathrm{~J} / \mathrm{kgK})$ & 130 \\
\hline (Goldsmith et al. [18]) & \\
\hline $\boldsymbol{k}(\mathrm{W} / \mathrm{mK})$ & 71.7 \\
$($ Yaws $[15])$ & \\
\hline
\end{tabular}


Table 3 Specifications of the adopted analytical/computational methodologies

\begin{tabular}{|c|c|c|c|}
\hline & $\begin{array}{c}\text { Carslaw and Jaeger } \\
\text { [1] }\end{array}$ & Paterson [21] & FLUENT \\
\hline Material Domain & $\begin{array}{c}a \rightarrow \infty \\
\text { (infinite) }\end{array}$ & $\begin{array}{c}0 \rightarrow \infty \\
\text { (infinite) }\end{array}$ & $\begin{array}{l}a \rightarrow b \\
\text { (finite) }\end{array}$ \\
\hline Wire Diameter & $2 a$ & 0 & $2 a$ \\
\hline No. of Phases & One Phase & $\begin{array}{c}\text { Two Phases } \\
\rho_{s}=\rho_{l}\end{array}$ & $\begin{array}{c}\text { Two Phases } \\
\rho_{s}=\rho_{l}\end{array}$ \\
\hline $\begin{array}{l}\text { Phase Change } \\
\text { Applicability }\end{array}$ & No & Yes & Yes \\
\hline $\begin{array}{c}\text { Pertinent } \\
\text { Dimensionless } \\
\text { Groups }\end{array}$ & $\begin{array}{c}\frac{T_{m}-T_{i}}{T_{i}} \\
\frac{q}{k T_{i}}\end{array}$ & $\begin{array}{c}\frac{T_{m}-T_{i}}{T_{i}}, \\
\frac{q}{k_{l} T_{i}}, \frac{\alpha_{l}}{\alpha_{s}}, \\
\text { Ste }=\frac{\left(C_{p}\right)_{l} T_{i}}{L}\end{array}$ & $\begin{array}{c}\frac{T_{m}-T_{i}}{T_{i}}, \\
\frac{q}{k_{l} T_{i}}, \frac{\alpha_{l}}{\alpha_{s}}, \\
\text { Ste }=\frac{\left(C_{p}\right)_{l} T_{i}}{L} \\
\frac{a}{b}, \frac{k_{s}}{k_{l}}, \\
\frac{\alpha_{w}}{\alpha_{l}}, \frac{k_{w}}{k_{l}}\end{array}$ \\
\hline
\end{tabular}


Table 4 Predicted thermal conductivity data versus the initial solid-state temperature $\left(T_{i}\right)$ of eicosane using the FLUENT model

\begin{tabular}{|c|c|c|c|}
\hline$T_{i}(K)$ & $\begin{array}{c}k_{s}(W / m K) \\
\text { (Stryker and } \\
\text { Sparrow [13]) }\end{array}$ & $\begin{array}{c}\left(k_{s}\right)_{\text {predicted }}(W / m K) \\
\text { FLUENT }\end{array}$ & $\begin{array}{c}\text { R } \boldsymbol{R}^{2} \text { Coefficients of } \\
\text { Determination of the } \\
\text { Least-Squares Curve Fits }\end{array}$ \\
\hline 308.3 & 0.42 & $\begin{array}{c}0.4250 \\
(1.19 \% \text { error })\end{array}$ & 1 \\
\hline 308.4 & 0.42 & $\begin{array}{c}0.4066 \\
(3.19 \% \text { error })\end{array}$ & 0.9675 \\
\hline 308.5 & 0.42 & $\begin{array}{c}0.3239 \\
(22.88 \% \text { error })\end{array}$ & 0.9243 \\
\hline 308.6 & 0.42 & $\begin{array}{c}0.2723 \\
(35.17 \% \text { error })\end{array}$ & 0.9312 \\
\hline 308.7 & 0.42 & $\begin{array}{c}0.1861 \\
(55.69 \% \text { error })\end{array}$ & 0.9215 \\
\hline 308.9 & 0.42 & $\begin{array}{c}0.1486 \\
(64.62 \% \text { error })\end{array}$ & 0.9523 \\
\hline
\end{tabular}




\section{$\underline{\text { List of Figures }}$}

Figure 1 Schematic diagram of the model of the thermal conductivity variation near the melting temperature

Figure 2 Schematic diagram of melting of an infinite medium with a zero-thickness line heat source positioned along the symmetry axis $(r=0)$

Figure 3 Transient temperature rise at the interface of the platinum wire-eicosane versus the heating time predicted by the FLUENT model and compared to the ideal THW theory by Carslaw and Jaeger [1]; Also shown are predictions of the FLUENT model with thermal conductivity of the wire set to 1000 times that of platinum

Figure $4 \quad$ Variations of (a) the transcendental function (Eq. 11) versus $\lambda$ and (b) the position of the liquid-solid interface versus heating time, corresponding to the melting problem of Paterson [21]

Figure 5 (a) Contour of temperature in both solid and liquid domains versus time and location, and (b) temperature rise curves on the wire surface versus heating time according to FLUENT along with temperature rise predictions near $r=0$ based on the model of Paterson [21]

Figure 6 Temperature rise on the surface of the wire versus heating time with an initial temperature of $T_{i}=306 \mathrm{~K}$ (solid eicosane) using the FLUENT model

Figure 7 Temperature rise on the surface of the wire versus heating time with an initial temperature of $T_{i}=313 \mathrm{~K}$ (liquid eicosane) using the FLUENT model

Figure 8 Variations of the temperature on the surface of the wire versus heating time for six cases with phase change subject to different initial temperatures

Figure 9 Predicted "effective" thermal conductivity values of eicosane as a function of the initial solid-state temperature using the FLUENT model 
Figure 10 Temperature rise on the surface of the wire versus heating time with an initial temperature of $T_{i}=308.3 \mathrm{~K}$ using the FLUENT model

Figure 11 Temperature rise on the surface of the wire versus heating time with an initial temperature of $T_{i}=308.4 \mathrm{~K}$ using the FLUENT model

Figure 12 Temperature rise on the surface of the wire versus heating time with an initial temperature of $T_{i}=308.5 \mathrm{~K}$ using the FLUENT model

Figure 13 Temperature rise on the surface of the wire versus heating time with an initial temperature of $T_{i}=308.6 \mathrm{~K}$ using the FLUENT model

Figure 14 Temperature rise on the surface of the wire versus heating time with an initial temperature of $T_{i}=308.7 \mathrm{~K}$ using the FLUENT model

Figure 15 Temperature rise on the surface of the wire versus heating time with an initial temperature of $T_{i}=308.9 \mathrm{~K}$ using the FLUENT model 


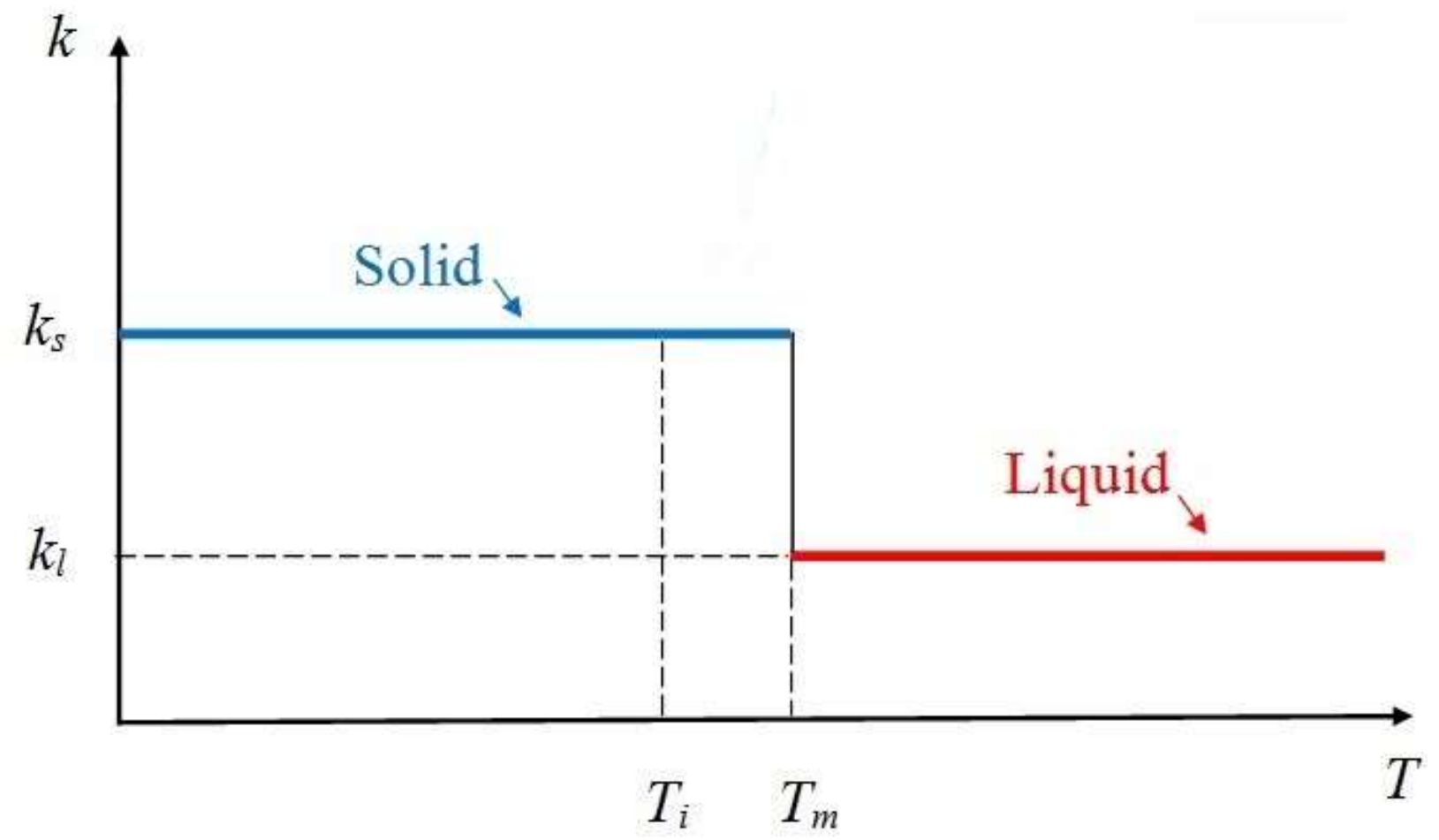

Figure 1 Schematic diagram of the model of the thermal conductivity variation near the melting temperature 


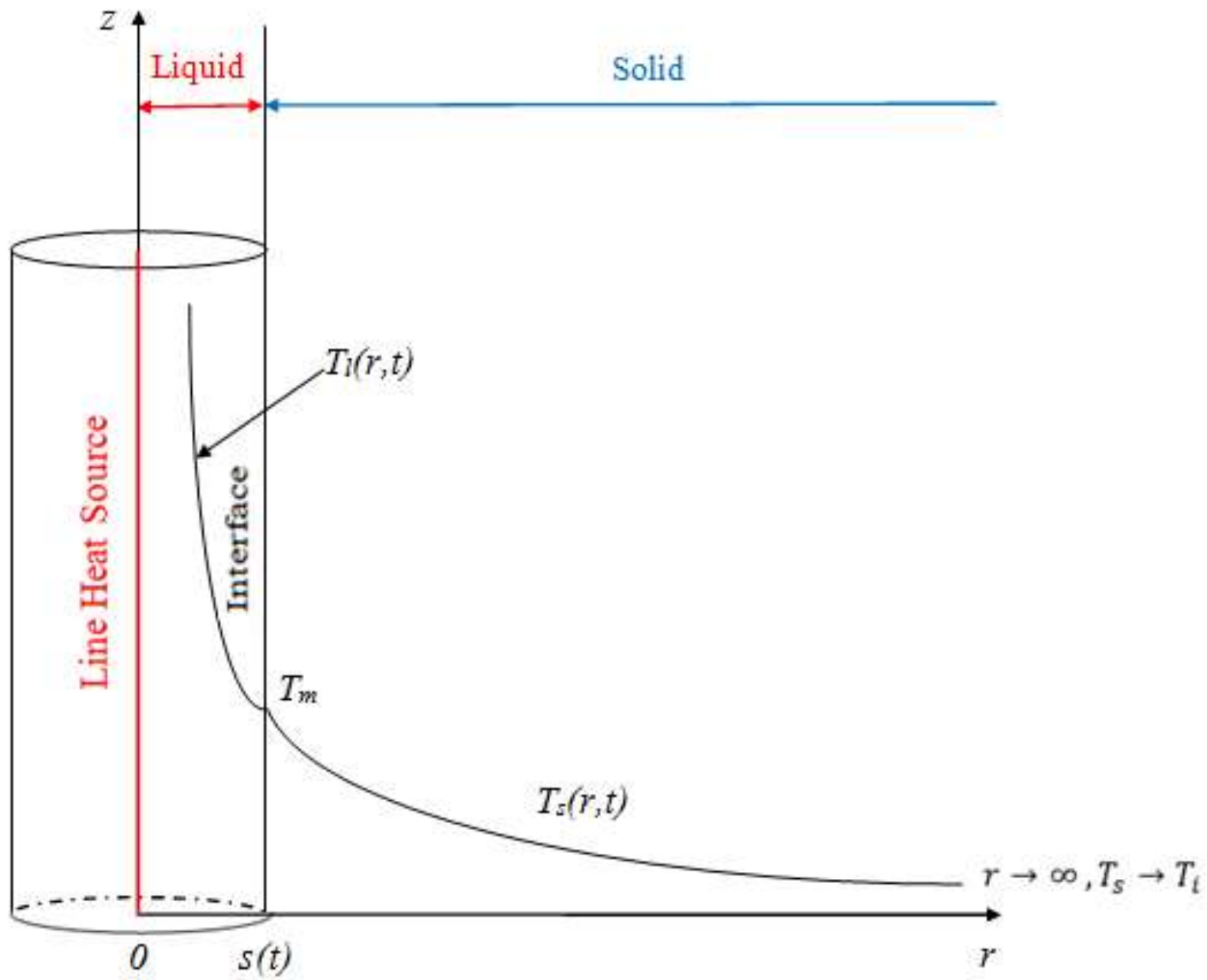

Figure 2 Schematic diagram of melting of an infinite medium with a zero-thickness line heat source positioned along the symmetry axis $(r=0)$ 


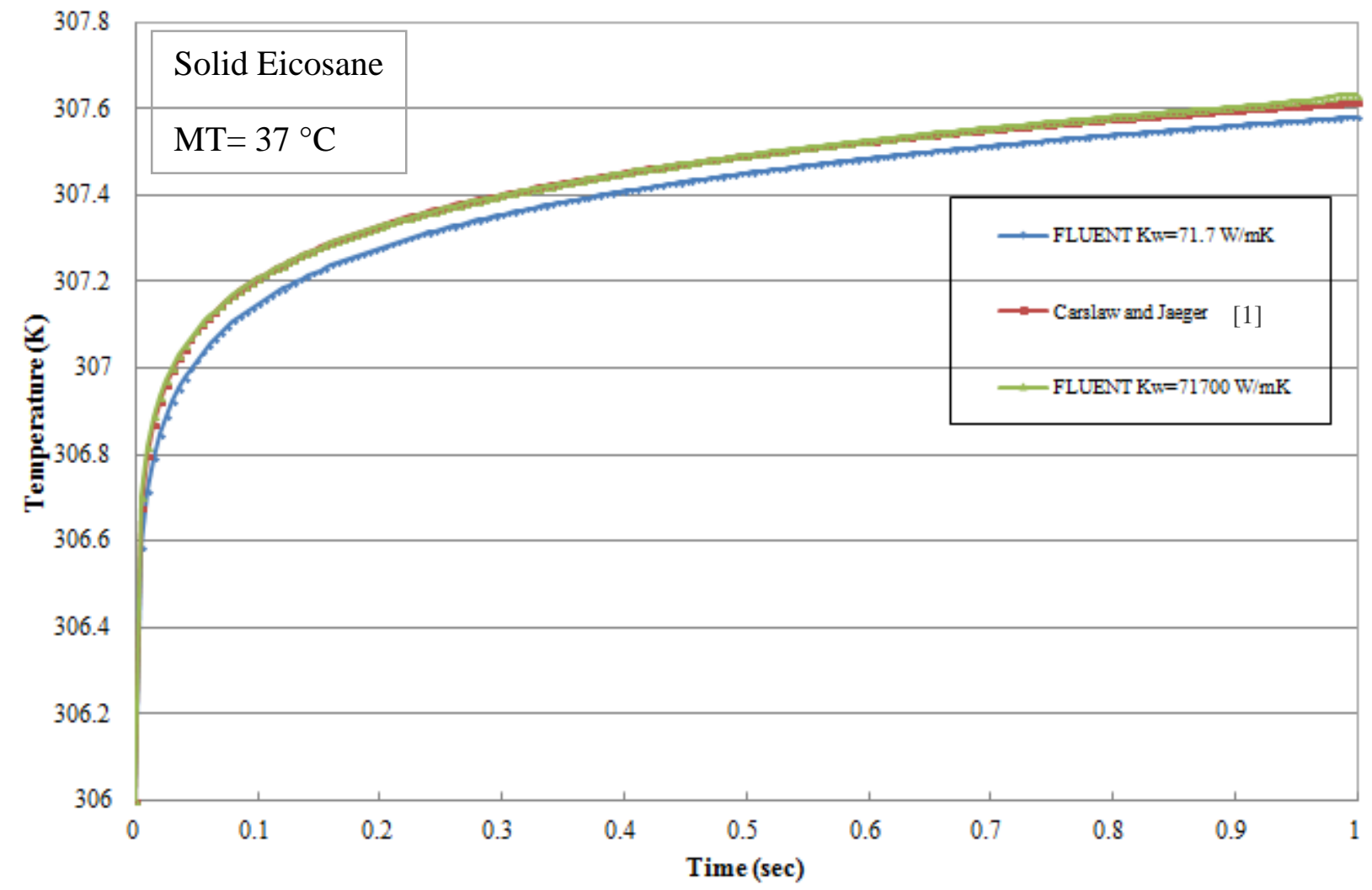

Figure 3 Transient temperature rise at the interface of the platinum wire-eicosane versus the heating time predicted by the FLUENT model and compared to the ideal THW theory by Carslaw and Jaeger [1]; Also shown are predictions of the FLUENT model with thermal conductivity of the wire set to 1000 times that of platinum 


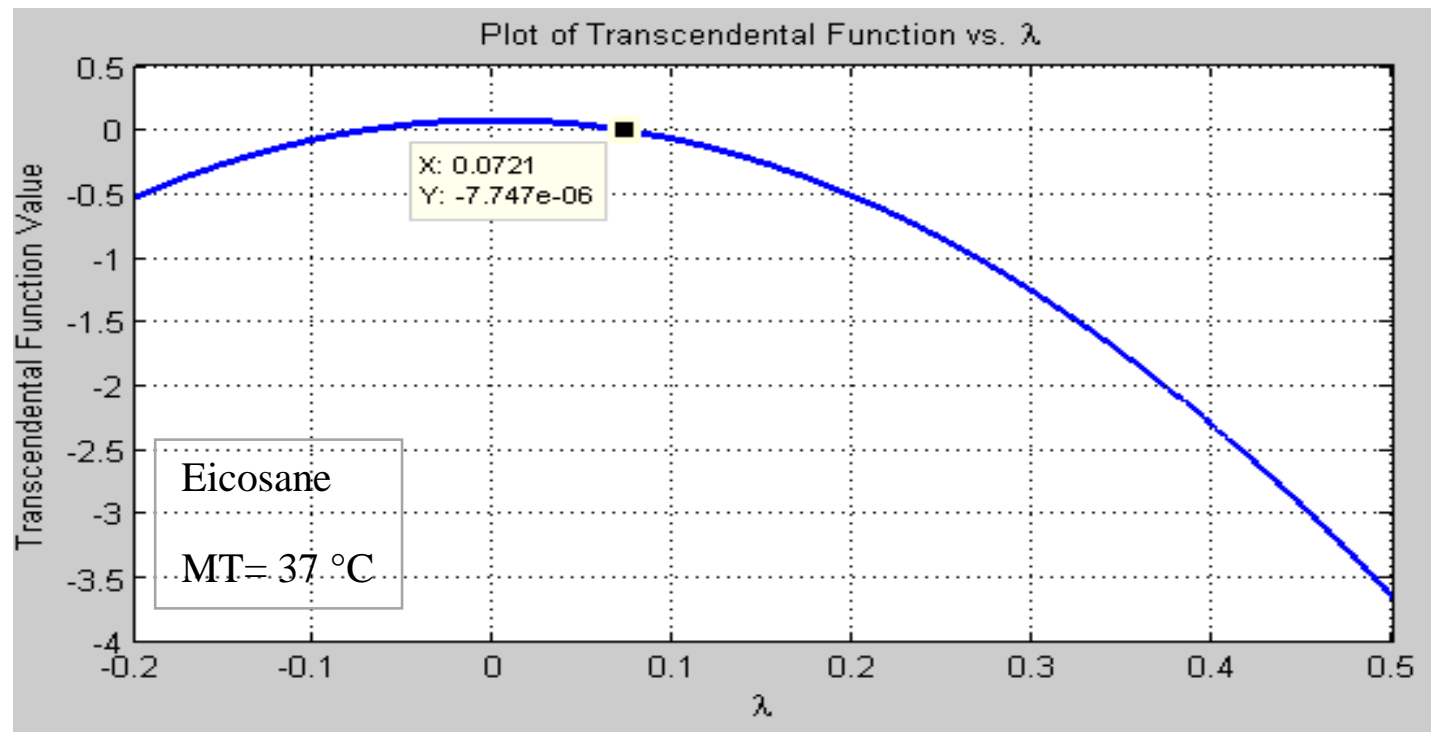

(a)

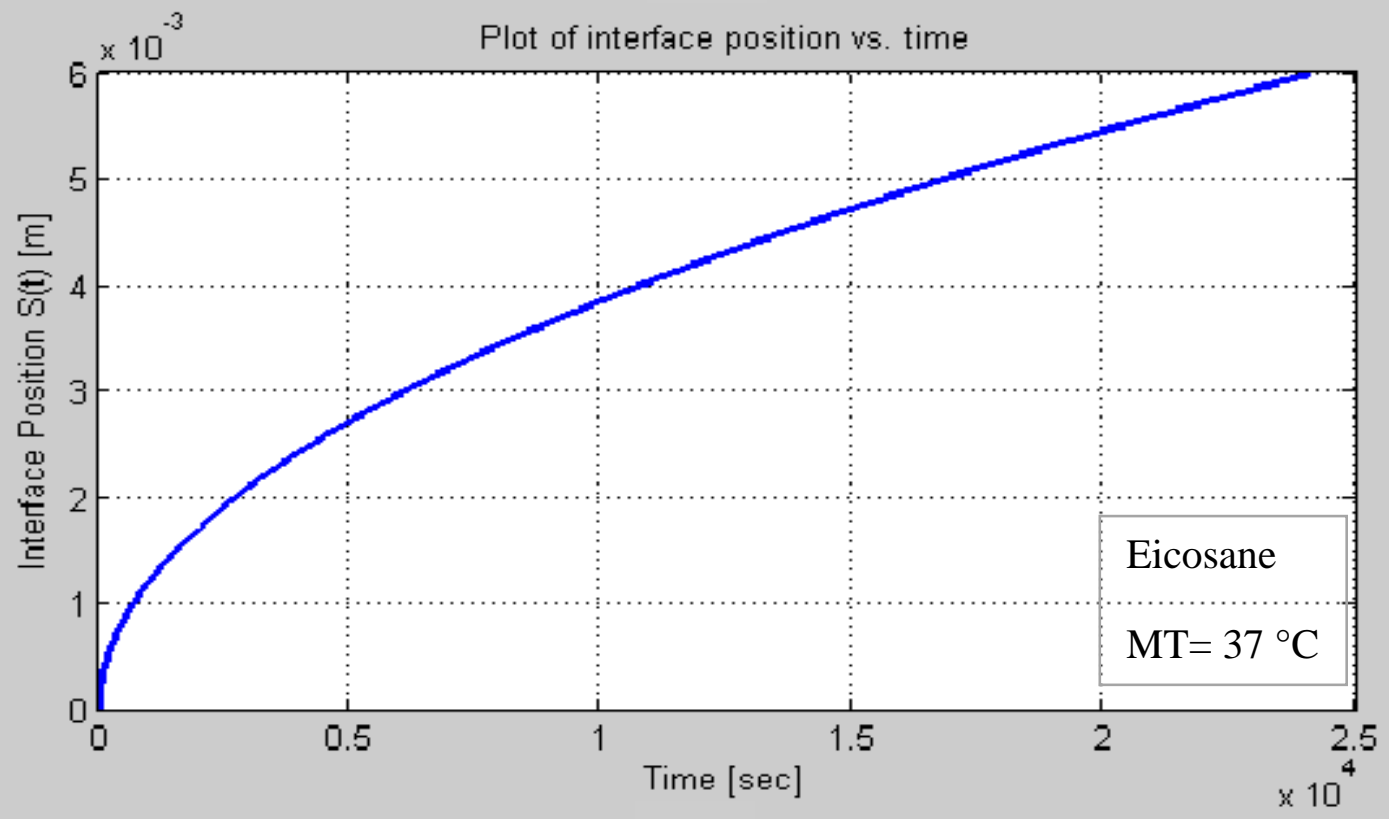

(b)

Figure 4 Variations of (a) the transcendental function (Eq. 11) versus $\lambda$ and (b) the position of the liquid-solid interface versus time, corresponding to the melting problem of Paterson [21] 


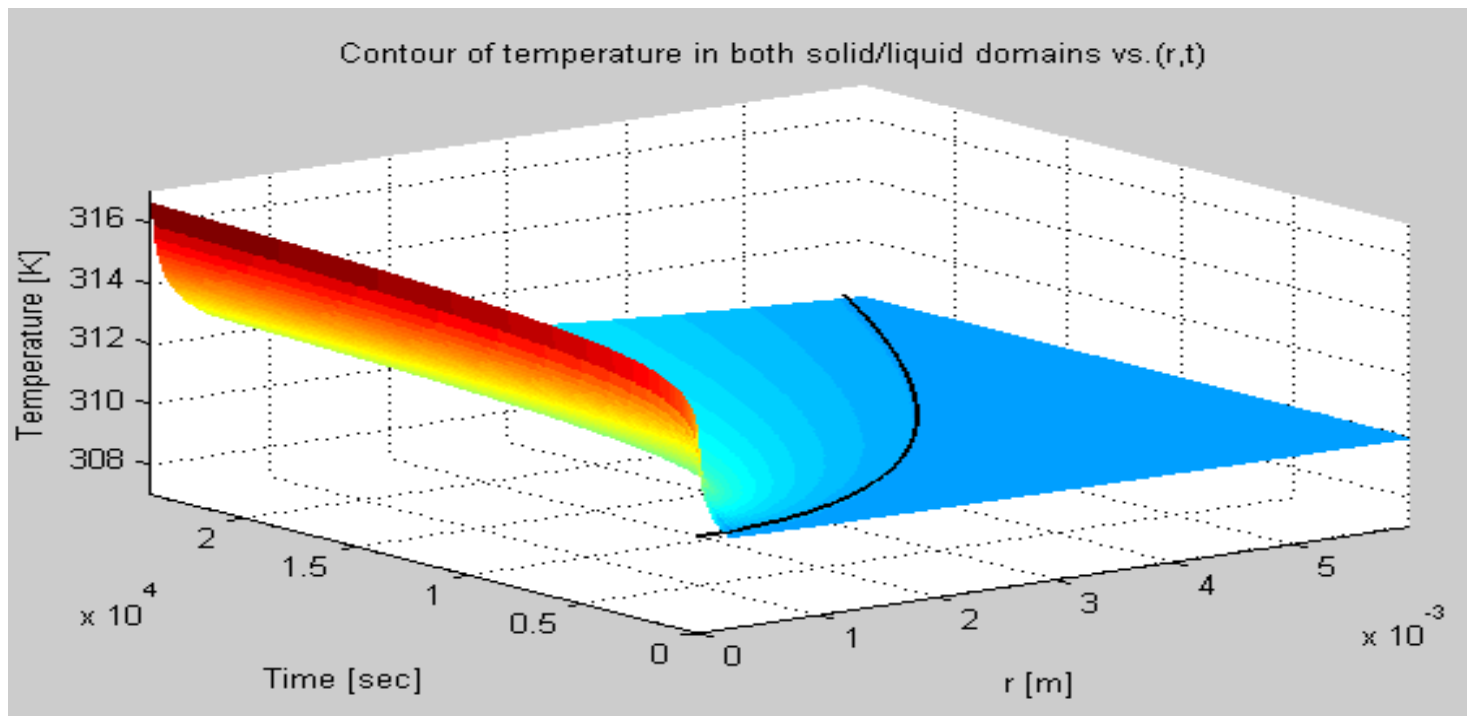

(a)

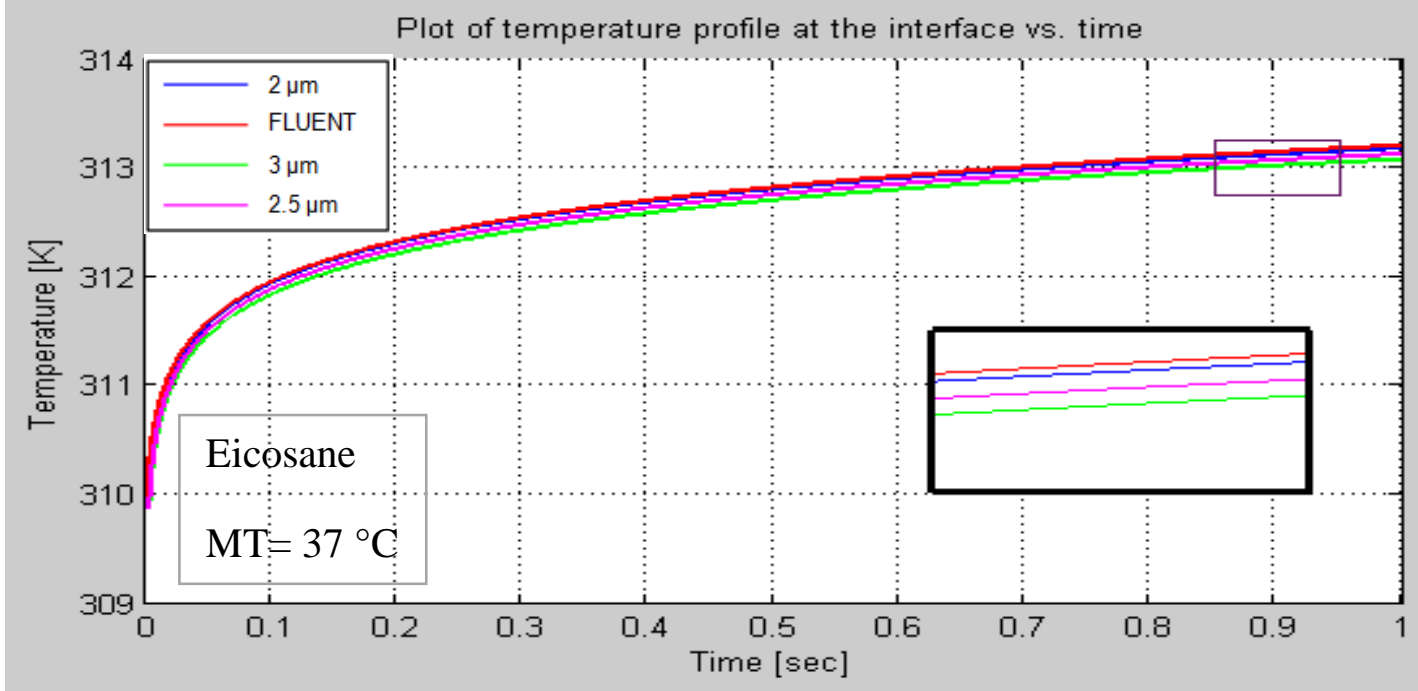

(b)

Figure 5 (a) Temperature distribution in both solid and liquid domains versus time and location, and (b) temperature rise curves on the wire surface versus time according to FLUENT along with temperature rise predictions near $r=0$ based on the model of Paterson [21] 


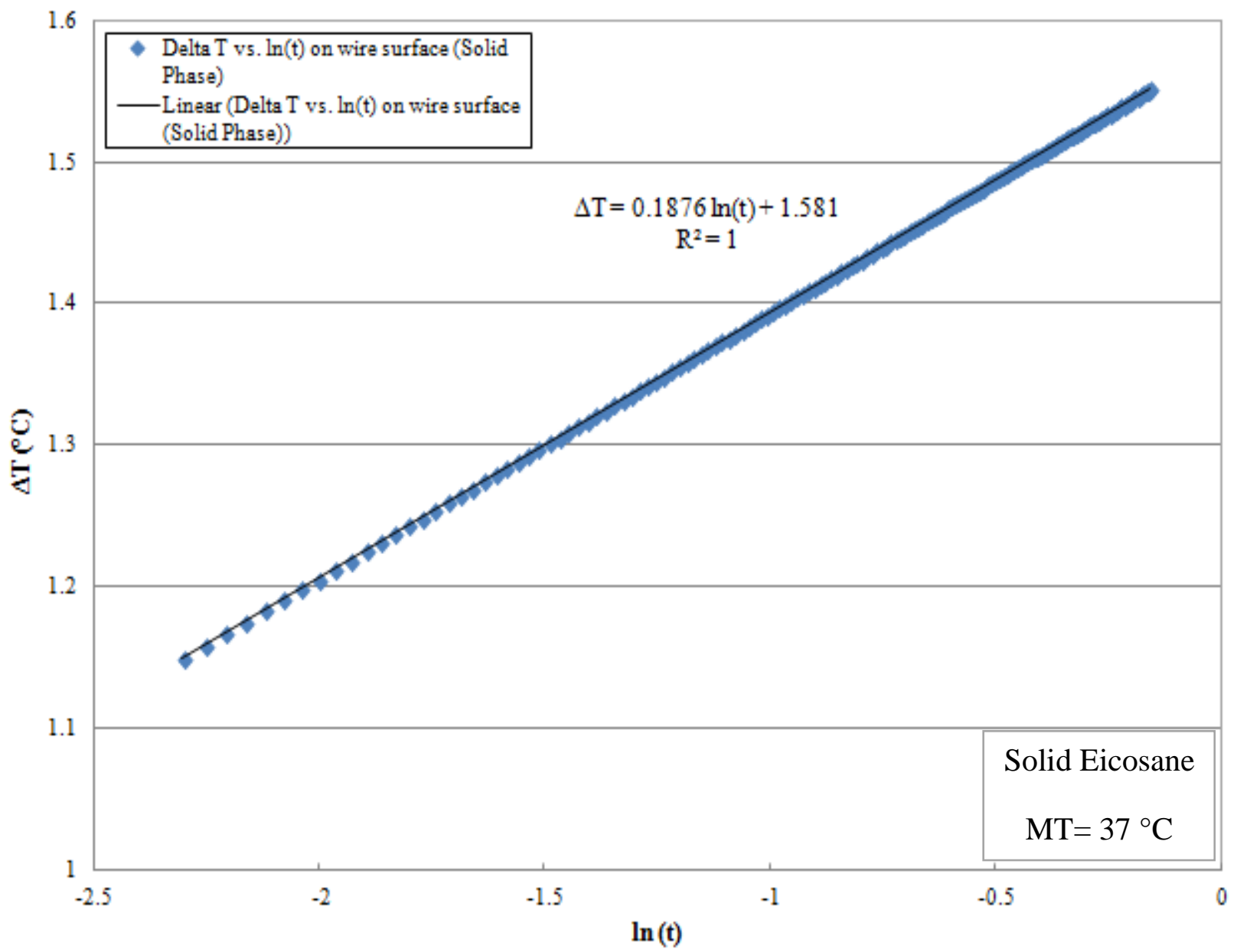

Figure 6 Temperature rise on the surface of the wire versus heating time with an initial temperature of $T_{i}=306 \mathrm{~K}$ (solid eicosane) using the FLUENT model 


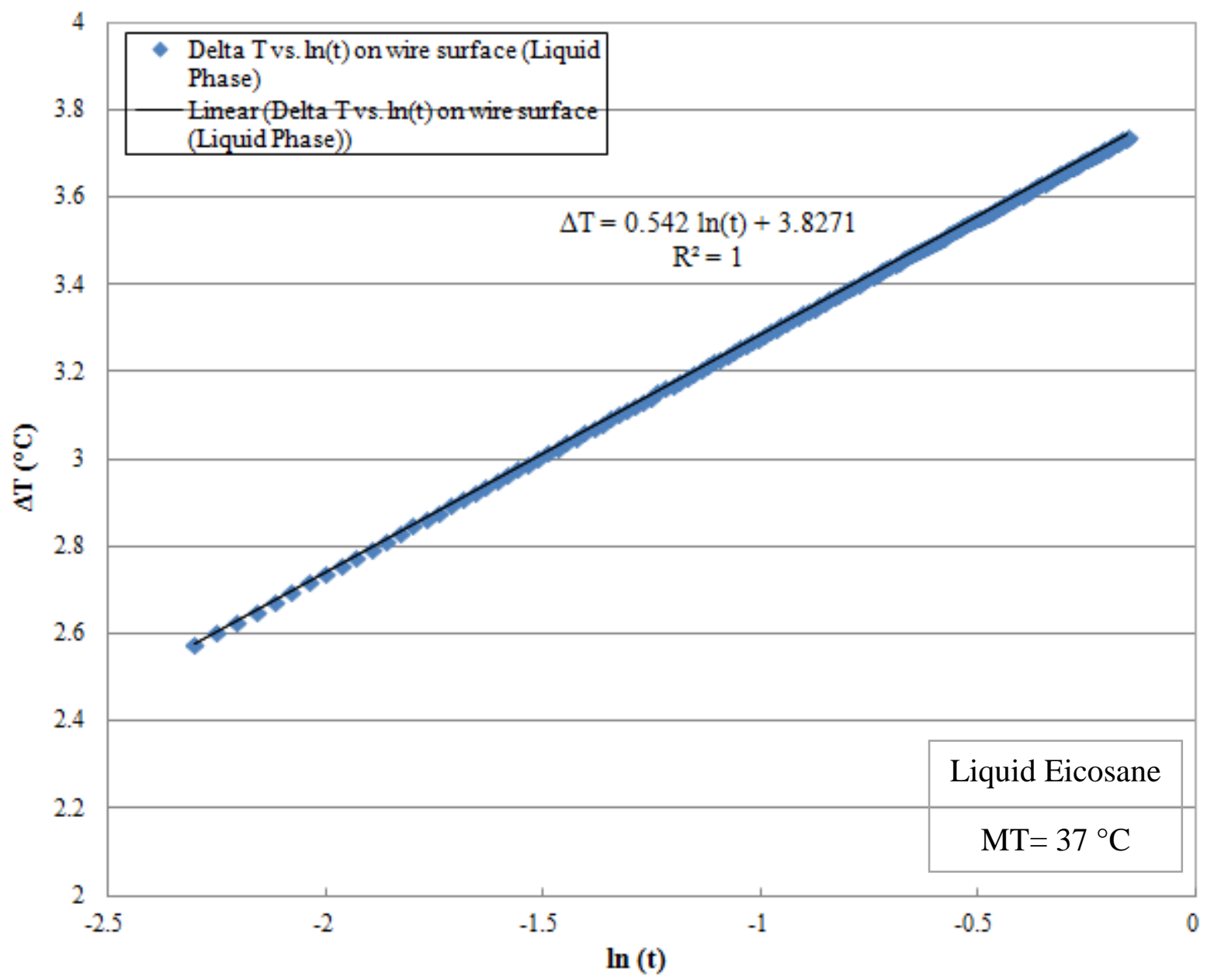

Figure 7 Temperature rise on the surface of the wire versus heating time with an initial temperature of $T_{i}=313 \mathrm{~K}$ (liquid eicosane) using the FLUENT model 


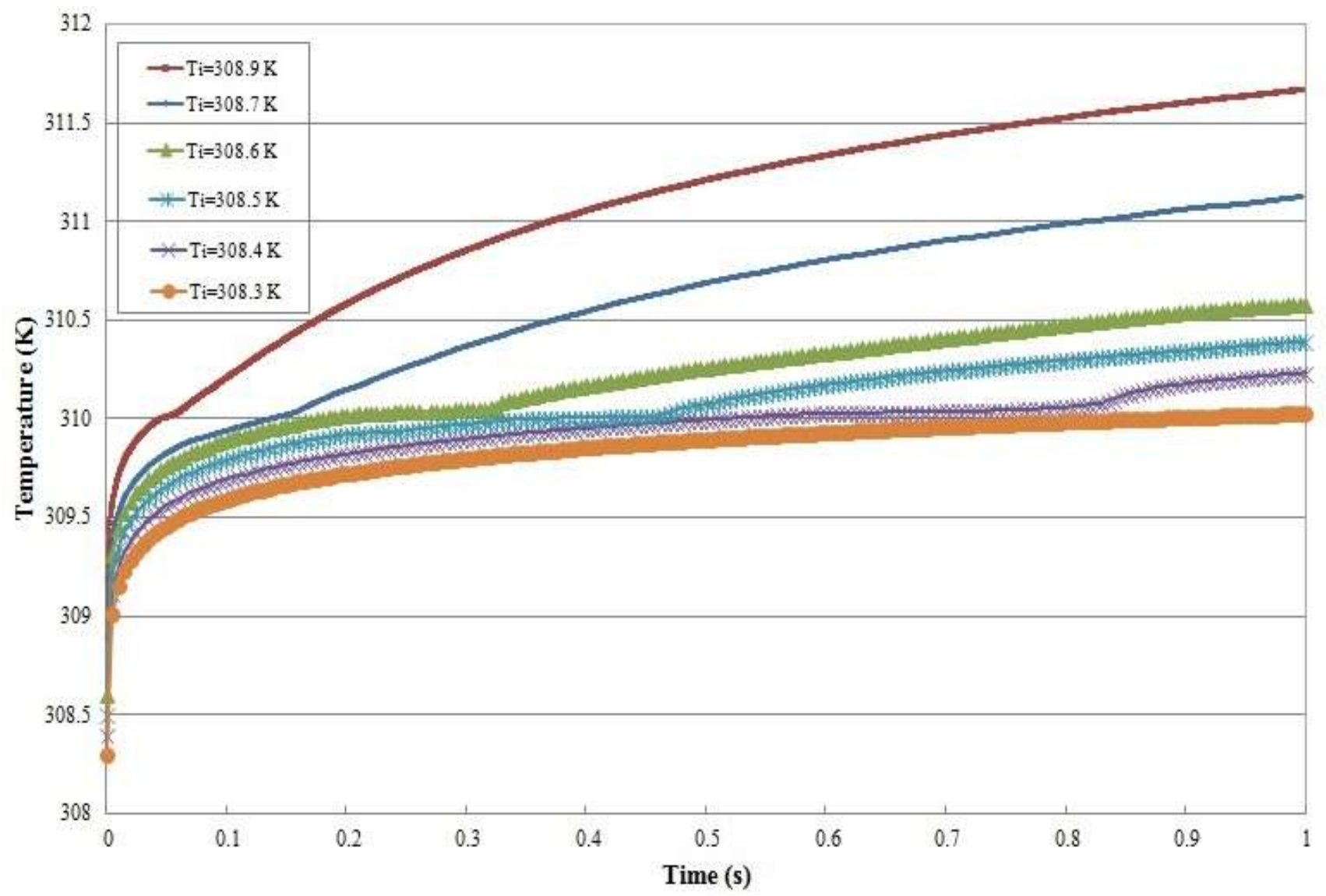

Figure 8 Variations of temperature on the surface of the wire versus heating time for six cases with phase change subject to different initial temperatures 


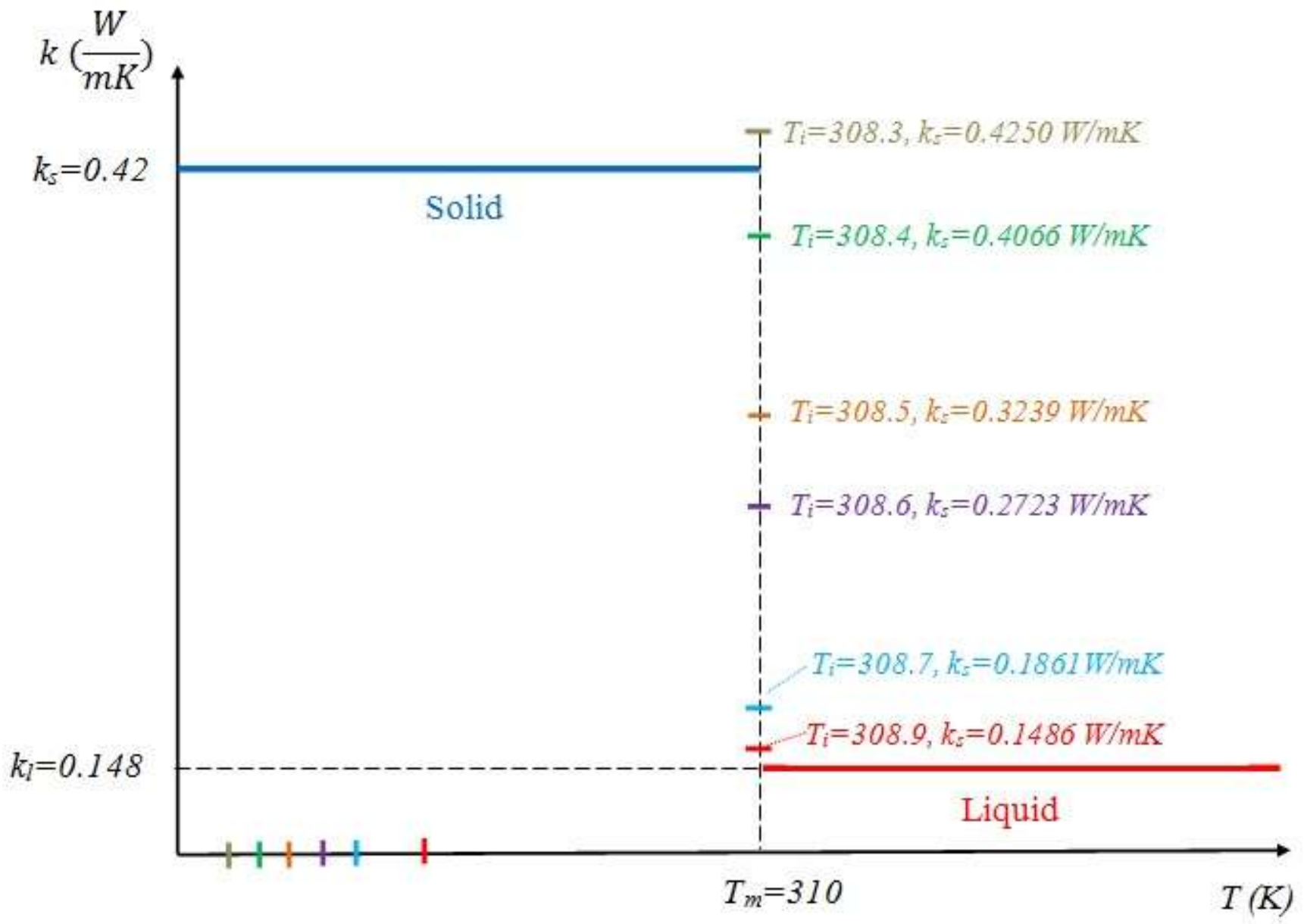

Figure 9 Predicted "effective" thermal conductivity values of eicosane as a function of the initial solid-state temperature using the FLUENT model 


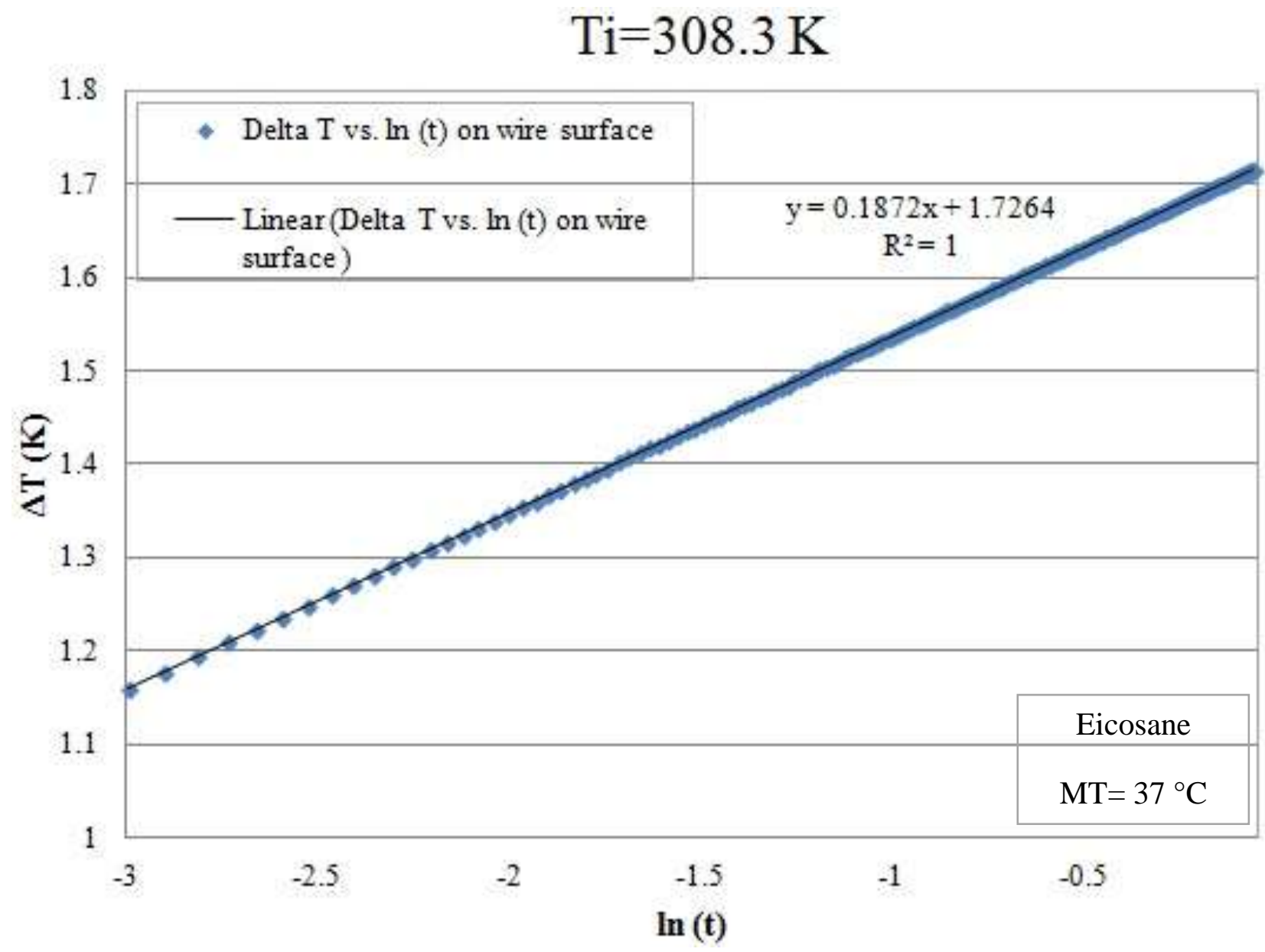

Figure 10 Temperature rise on the surface of the wire versus heating time with an initial temperature of $T_{i}=308.3 \mathrm{~K}$ using the FLUENT model 


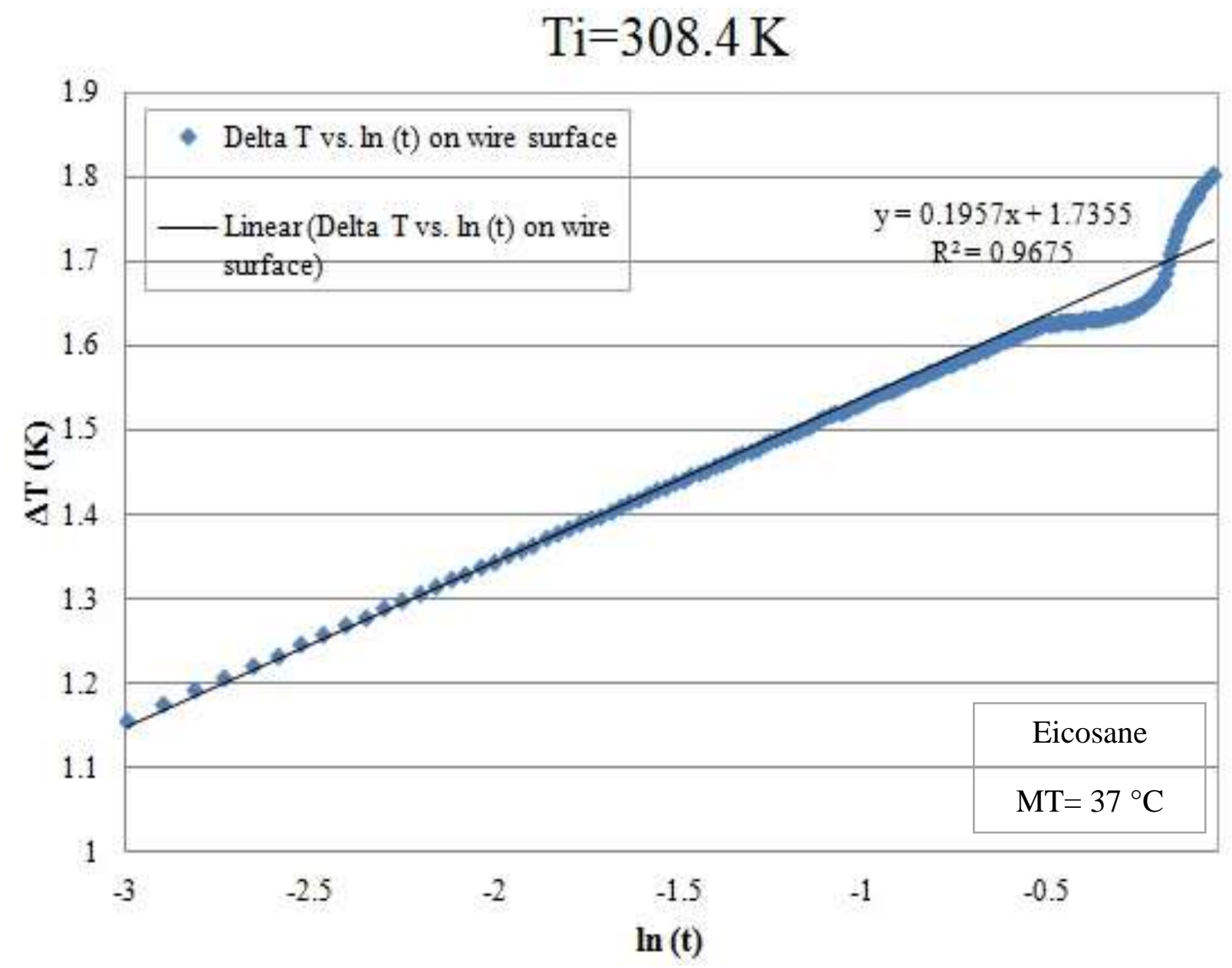

Figure 11 Temperature rise on the surface of the wire versus heating time with an initial temperature of $T_{i}=308.4 \mathrm{~K}$ using the FLUENT model 


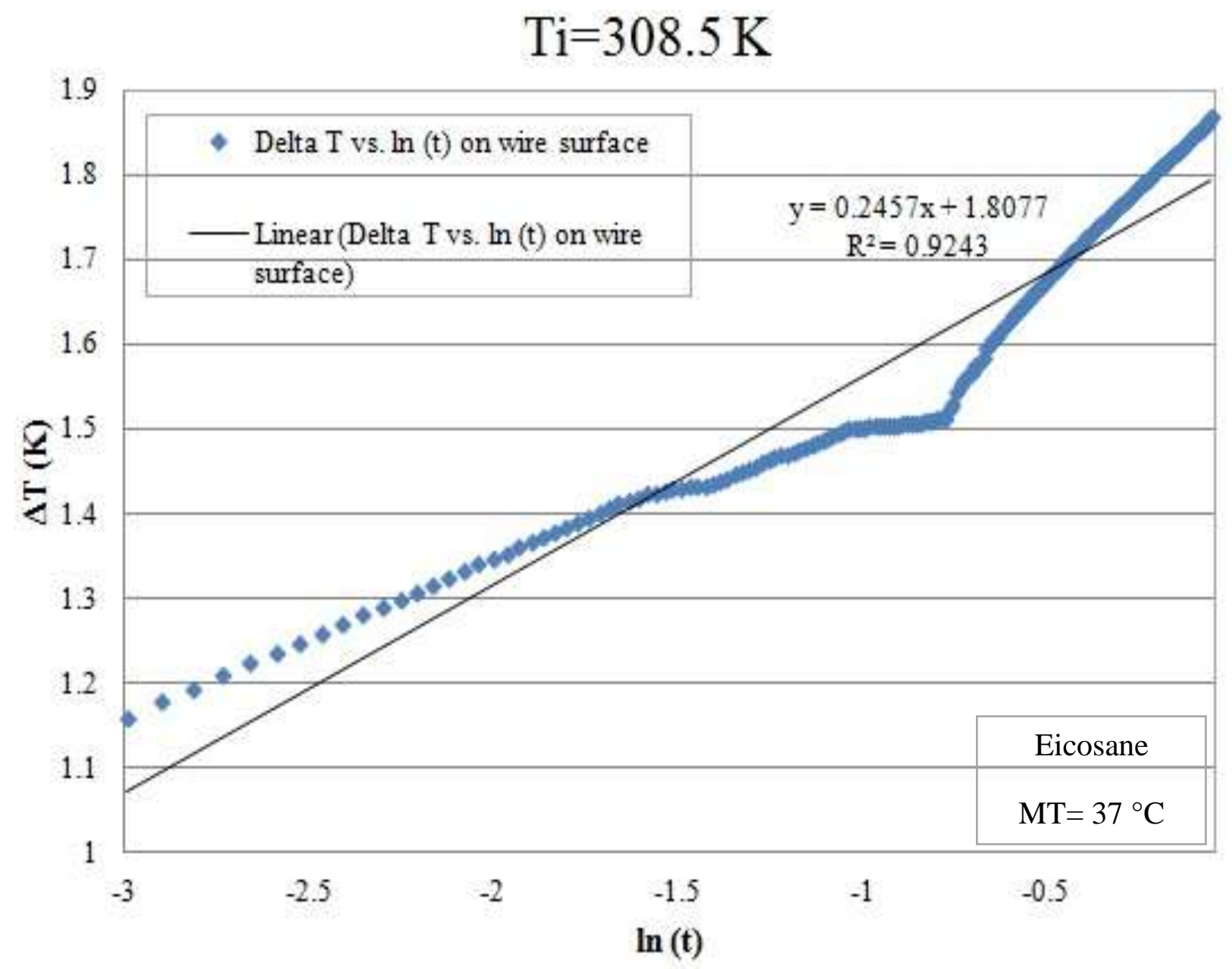

Figure 12 Temperature rise on the surface of the wire versus heating time with an initial temperature of $T_{i}=308.5 \mathrm{~K}$ using the FLUENT model 


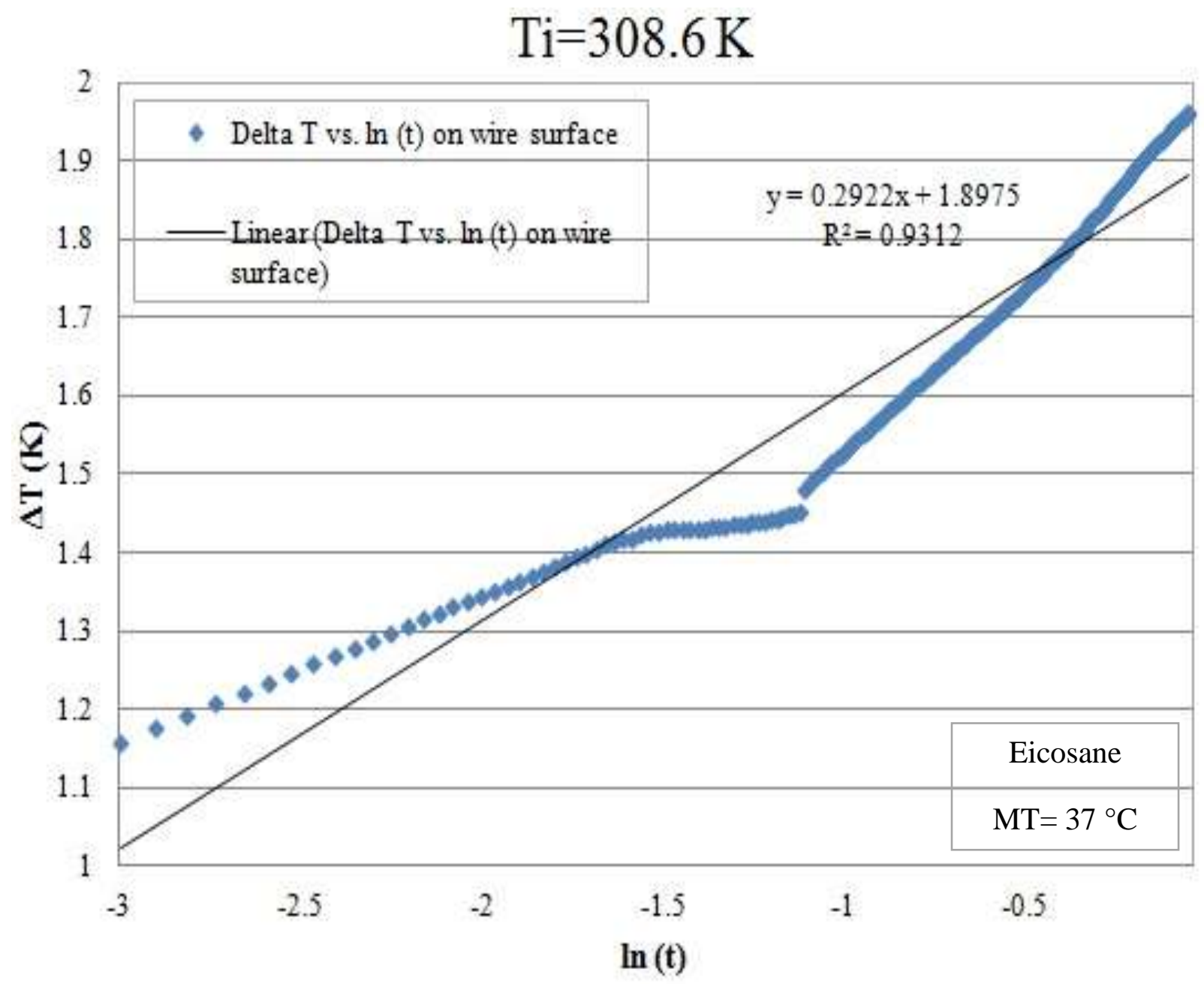

Figure 13 Temperature rise on the surface of the wire versus heating time with an initial temperature of $T_{i}=308.6 \mathrm{~K}$ using the FLUENT model 


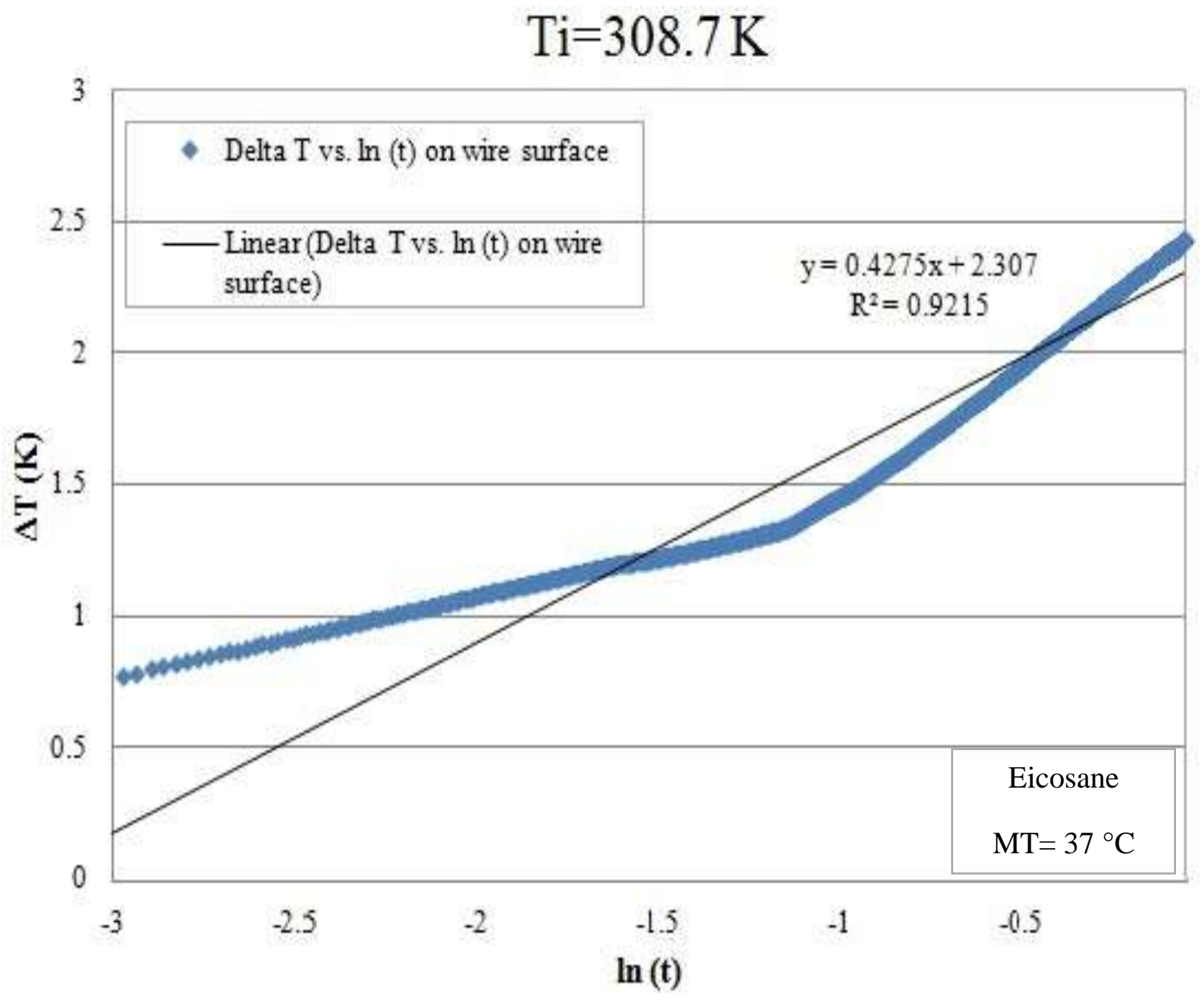

Figure 14 Temperature rise on the surface of the wire versus heating time with an initial temperature of $T_{i}=308.7 \mathrm{~K}$ using the FLUENT model 


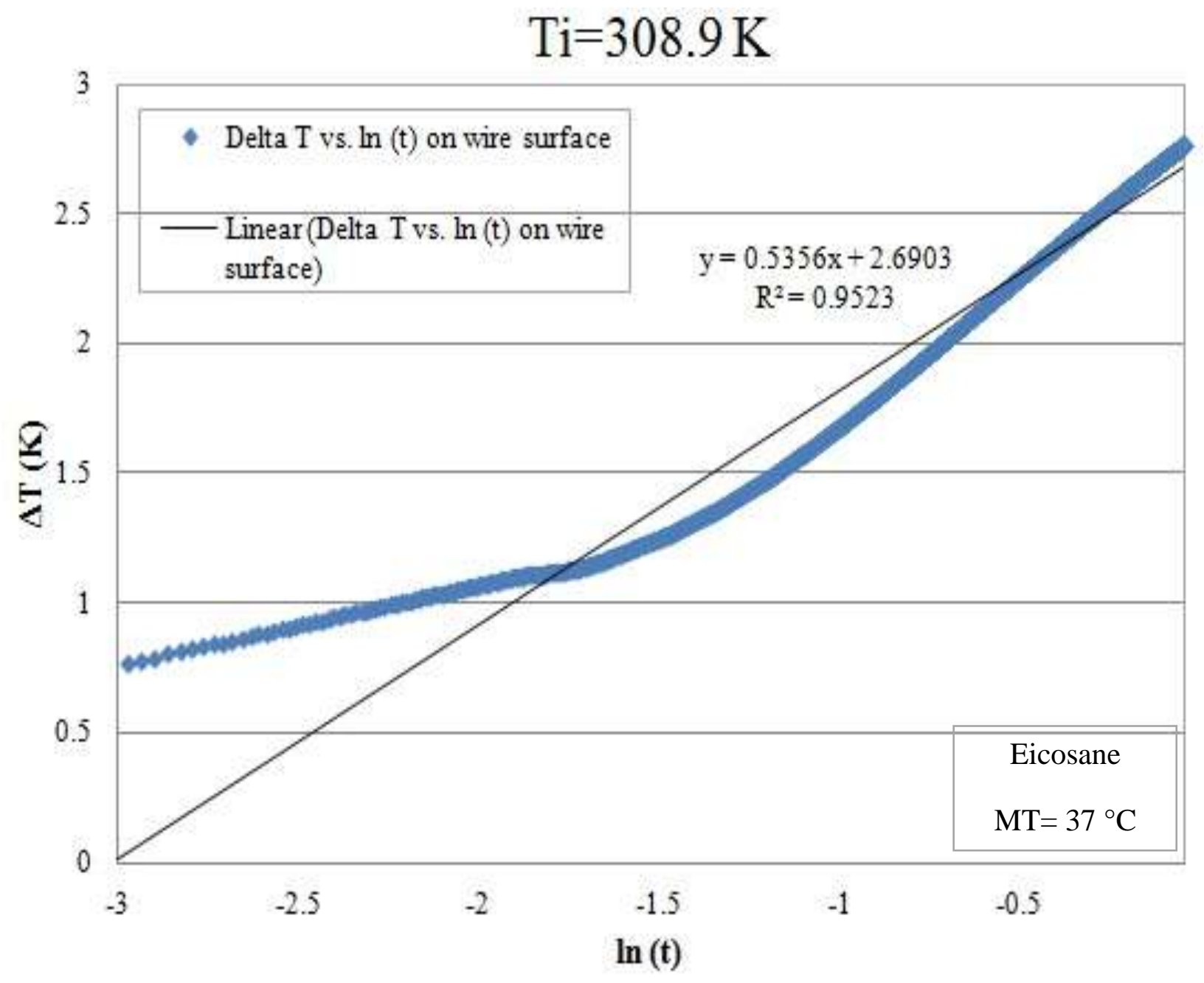

Figure 15 Temperature rise on the surface of the wire versus heating time with an initial temperature of $T_{i}=308.9 \mathrm{~K}$ using the FLUENT model 